\title{
A generalized few-state model for the first hyperpolarizability
}

\author{
Md Mehboob Alam, ${ }^{1, \text { a) }}$ Maarten T. P. Beerepoot, ${ }^{2}$ and Kenneth Ruud ${ }^{2, b)}$ \\ 1) Department of Chemistry, Indian Institute of Technology Bhilai. GEC campus, Sejbahar, Raipur, Chhattisgarh - 492015, \\ India. \\ ${ }^{2)}$ The Hylleraas Centre for Quantum Molecular Sciences, Department of Chemistry, University of Troms $\phi-$ The Arctic University \\ of Norway, Troms $\phi$, Norway
}

(Dated: 2 June 2020)

The properties of molecules depend on their chemical structure and thus structure-property relations help design molecules with desired properties. Few-state models are often used to interpret experimental observations of nonlinear optical properties. Not only the magnitude, but also the relative orientation of the transition dipole moment vectors is needed for few-state models of the non-linear optical properties. The effect of the relative orientation of the transition dipole moment vectors is called dipole alignment and this effect has previously been studied for multiphoton absorption properties. However, so far no such studies are reported for the first hyperpolarizability. Here we present a generalized few-state model for the static and dynamic first hyperpolarizability $\beta$, accounting for the effect of dipole alignment. The formulae derived in this work are general in the sense that they can be used for any few-state model, i.e. two-state model, three-state model or in general an $n$-state model. Based on the formulae, we formulate minimization and maximization criteria for the alignment of transition dipole moment vectors. We demonstrate the importance of dipole alignment by applying the formulae to the static first hyperpolarizability of ortho-, meta- and para-nitroaniline. The formulae and the analysis provide new ways to understand structure-property relationship for $\beta$ and can hence be used to fine-tune the magnitude of $\beta$ in a molecule.

\section{INTRODUCTION}

State-of-the-art photonic applications such as bioimaging, ${ }^{1}$ photodynamic therapy, ${ }^{2,3}$ electro-optical devices, ${ }^{4}$ and three-dimensional data storage ${ }^{5}$ depend on the non-linear optical properties of the electronic (atomic/molecular/ionic) systems involved. A system exhibits non-linear optical properties when the applied electric field is sufficiently strong so that the corresponding polarization no longer varies linearly with the strength of the field. ${ }^{6,7}$ The first hyperpolarizability $(\beta)$ is related to the second-order susceptibility and quantifies the change in the dipole moment induced by an electric field. Being a second-order non-linear optical property, $\beta$ vanishes for centro-symmetric systems. A static electric field can be used to break the inversion symmetry of the macroscopic system and allow $\beta$ to be measured in electric-field induced second-harmonic generation (EFISHG) experiments. ${ }^{8-10}$

In general, $\beta$ involves three different frequencies, two of which are the frequencies of the incident optical fields and the remaining one is the frequency of the resulting optical field. Hence $\beta$ is written as $\beta\left(-\omega_{3} ; \omega_{1}, \omega_{2}\right)$ subject to the condition $\omega_{1}+\omega_{2}=\omega_{3}$. In the static case, all three frequencies are zero and hence $\beta_{\text {static }} \equiv \beta(0 ; 0,0)$. Depending on the incident frequencies, several properties related to $\beta$ have been realized experimentally ${ }^{7}$ such as sum-frequency generation $(\mathrm{SHG})^{11}\left[\beta\left(-\omega_{1}-\omega_{2} ; \omega_{1}, \omega_{2}\right)\right]$, difference-frequency generation $^{12}\left[\beta\left(-\omega_{1}+\omega_{2} ; \omega_{1},-\omega_{2}\right)\right]$, electro-optical Pockels effect $^{13}[\beta(-\omega ; \omega, 0)]$, optical rectification ${ }^{14}[\beta(0 ; \omega,-\omega)]$, and second-harmonic generation ${ }^{15}[\beta(-2 \omega ; \omega, \omega)]$. The current interest in systems that have non-linear optical properties focuses on the development of systems with large values of $\beta$

\footnotetext{
a)Electronic mail: mehboob@iitbhilai.ac.in

b)Electronic mail: kenneth.ruud@uit.no
}

as well as on fine-tuning $\beta$ in different systems. Several experimental and theoretical works have contributed to these goals. For example, the effect of $\pi$-conjugation, ${ }^{16-18}$ bond-length alternation, ${ }^{19-21}$ and solvent ${ }^{22,23}$ have been explored experimentally and/or theoretically.

Sum-over-states (SOS) expressions are often used for the theoretical calculations of $\beta^{24-31}$ and can be extracted from time-dependent perturbation theory for exact states. ${ }^{32,33}$ This approach defines different components of $\beta$ in terms of transition dipole moment vectors (TDMVs) and excitation energies of all excited states of the system and is thus computationally expensive. In addition to excitation energies and ground- to excited-state transition dipole moments (TDMs), the approach also requires TDMs for excitations between different excited states. In many cases, however, only a few states contribute strongly to the observed non-linear optical response, and the full SOS expression is often truncated to a few essential states, giving rise to so-called few-state models. The popularity of few-state models is due to their simplicity and their ability to reproduce the experimental results qualitatively. Few-state models are especially useful for push-pull $\pi$-conjugated systems with one dominant charge transfer direction. One of the limitations of the few-state or SOS models, however, is that the direction of the TDMVs does not appear in the expressions that are usually used. Thus, the effect of the relative orientation of the TDMVs - also called dipole alignment - on $\beta$ cannot be explicitly studied using standard SOS and few-state models. Since the relative orientation of TDMVs are directly related to the structure of the molecules (e.g. to the position of electron-attracting or electron-donating groups), the exploration of the effect of these orientations on $\beta$ can provide new ways to understand the corresponding structure-property relationships. The effect of the relative orientation of TDMVs on the two-, three- and in general multiphoton absorption properties has been explored by Cronstrand et al. ${ }^{34}$ and Alam et $a l .{ }^{35-38}$ Alam et al. have also studied the effect of solvent and 
geometry on the orientation of the TDMVs in multiphoton absorption properties. ${ }^{39-41}$ These studies have demonstrated the importance of dipole alignment in multiphoton absorption processes. Even though the vector nature of the TDMVs has been taken into account in some works, ${ }^{42,43}$ no such explicit studies have been performed for $\beta$ as far as we know. We here fill this gap by presenting generalized few-state model formulae including the effect of dipole alignment on the static and dynamic $\beta$. We apply the derived formulae on the wellknown simple molecules ortho-, meta- and para-nitroaniline to demonstrate the importance of dipole alignment.

The manuscript is organized as follows: in Section II, the basic theory and formulae for $\beta$ are described. In Section III, the generalized few-state model formulae for different types of $\beta$ are derived. This is followed by some explicit expressions for different few-state models in Section IV. The application of the derived formulae is illustrated in Section VI followed by concluding remarks in Section VII.

\section{BASIC THEORY FOR $\beta$}

$\beta$ can be defined in terms of the response of the energy to an externally applied electric field as ${ }^{44,45}$

$$
\begin{aligned}
E(F) & =E(0)-\sum_{i} \mu_{i} F_{i}-\frac{1}{2 !} \sum_{i, j} \alpha_{i j} F_{i} F_{j} \\
& -\frac{1}{3 !} \sum_{i, j, k} \beta_{i j k} F_{i} F_{j} F_{k}-\frac{1}{4 !} \sum_{i, j, k, l} \gamma_{i j k l} F_{i} F_{j} F_{k} F_{l}-\ldots,
\end{aligned}
$$

where $E(F), \mu_{i}(F), \alpha_{i j}(F), \beta_{i j k}$ and $\gamma_{i j k l}$ are the energy of the system, the $i$-th component of the dipole moment vector, the $i j$-th element of the second-rank polarizability tensor, the $i j k$ th element of the third-rank first hyperpolarizability tensor, and the $i j k l$-th element of the fourth-rank second hyperpolarizability tensor, respectively, in the presence of an external electric field $(F)$. The components of the electric field are represented by $F_{i}, F_{j}, F_{k}, F_{l}$. Descriptions of different conventions and definitions used for defining $\beta$ can be found in the literature. ${ }^{44,46,47}$

The general SOS expression for the $i j k$-th component of the electronic $\beta\left(-\omega_{\xi} ; \omega_{1}, \omega_{2}\right)$ can be derived from timedependent perturbation theory and is given as ${ }^{24}$

$$
\beta_{i j k}\left(-\omega_{\xi} ; \omega_{1}, \omega_{2}\right)=\sum \mathscr{P}_{-\xi, 1,2} \sum_{P, Q}^{\prime} \frac{\mu_{i}^{O P} \bar{\mu}_{j}^{P Q} \mu_{k}^{Q 0}}{\left(E_{0 P}-E_{\xi}\right)\left(E_{0 Q}-E_{2}\right)},
$$

where $\omega_{1}, \omega_{2}$ and $\omega_{\xi}$ are the frequencies of the three optical fields involved, $E_{i}=\hbar \omega_{i}(i=1,2, \xi)$ and $\sum \mathscr{P}_{-\xi, 1,2}$ represents the summation over all the permutations of the pairs $\left(i,-\omega_{\xi}\right),\left(j, \omega_{1}\right)$ and $\left(k, \omega_{2}\right)$. Thus, $\sum \mathscr{P}_{-\xi, 1,2}$ represents a sum of six terms. The prime over the double summation $\left(\sum_{P, Q}^{\prime}\right)$ indicates the omission of the ground state from the summation, i.e., $P \neq 0, Q \neq 0$. $E_{P Q}$ and $\hat{\mu}_{k}^{P Q}$ are the excitation energy and the $k$-th component of the TDMV, respectively, for transition $|P\rangle \rightarrow|Q\rangle$. Finally, $\bar{\mu}=\mu-\mu^{00}$. Using the components $\beta_{i j k}$, one can define the total $\beta\left(\beta_{\text {tot }}\right)$ as,

$$
\beta_{\mathrm{tot}}=\sqrt{\beta_{x}^{2}+\beta_{y}^{2}+\beta_{z}^{2}}=\sqrt{\sum_{i=x, y, z} \beta_{i}^{2}}
$$

with

$$
\beta_{i}=\frac{1}{5} \sum_{j=x, y, z}\left(\beta_{i j j}+\beta_{j i j}+\beta_{j j i}\right) .
$$

One can also define the vector quantity $\beta_{\mathrm{vec}}$, which is the vector component of $\beta$ in the direction of the dipole moment. This quantity is useful in comparing the value of $\beta$ obtained in EFISHG experiments ${ }^{8-10}$ on polar molecules and is given as

$$
\beta_{\mathrm{vec}}=\sum_{i=x, y, z} \frac{\mu_{i} \beta_{i}}{|\mu|}
$$

where $|\mu|$ is the ground-state dipole moment of the system with $\mu=\sqrt{\sum_{i=x, y, z} \mu_{i}^{2}}$ and $\beta_{i}$ is defined in Eq. 4. The isotropically averaged parallel $\left(\beta_{\|}\right)$and perpendicular $\left(\beta_{\perp}\right) \beta$ s are given as

$$
\begin{aligned}
& \beta_{\|}=\frac{1}{5} \sum_{i, j}\left(\beta_{i j j}+\beta_{j i j}+\beta_{j j i}\right) \bar{\mu}_{i}^{00} \\
& \beta_{\perp}=\frac{1}{5} \sum_{i, j}\left(2 \beta_{i j j}-3 \beta_{j i j}+2 \beta_{j j i}\right) \bar{\mu}_{i}^{00}
\end{aligned}
$$

where $\bar{\mu}_{i}^{00}$ represents the ground-state dipole unit in the direction of the ground-state dipole moment. It is equal to $\frac{\mu_{i}^{00}}{\mu^{00}}, \mu_{i}^{00}$ being the $i$-th component of the ground-state dipole moment vector. Thus, $\beta_{\text {vec }}=\beta_{\|}$.

From Eq. 2 one can observe that each component of $\beta$ involves three TDMVs. Since these TDMVs are vector quantities, both $\beta_{\mathrm{ijk}}$ and $\beta$ depend not only on the magnitudes of these vectors but also on their relative orientation. However, in the final expressions for $\beta$ (Eqs. 5, 6a and $6 \mathrm{~b}$ ), only the magnitudes of the different components of $\left(\beta_{\mathrm{ijk}}\right)$ and hence those of the TDMVs are used. The value of $\beta$ calculated using the above equations has been reported in several instances, ${ }^{24-31}$ but the vector nature of the TDMVs have never been explored. In the next section, we derive the contributions from the relative orientations of TDMVs on the expressions for $\beta$.

\section{DERIVATION OF GENERALIZED FEW-STATE MODEL FOR $\beta$ : EFFECT OF DIPOLE ALIGNMENT}

The first step in the derivation of a generalized few-state model for $\beta$ is to expand the permutation operator $\mathscr{P}_{-\xi, 1,2}$ in Eq. 2, giving an expression for $\beta_{i j k}$ with six terms as 


$$
\begin{aligned}
\beta_{i j k}\left(-\omega_{\xi} ; \omega_{1}, \omega_{2}\right)=\sum_{P, Q}^{\prime} & {\left[\frac{\mu_{i}^{0 P} \bar{\mu}_{j}^{P Q} \mu_{k}^{Q 0}}{\left(E_{0 P}-E_{\xi}\right)\left(E_{0 Q}-E_{2}\right)}+\frac{\mu_{i}^{0 P} \bar{\mu}_{k}^{P Q} \mu_{j}^{Q 0}}{\left(E_{0 P}-E_{\xi}\right)\left(E_{0 Q}-E_{1}\right)}+\frac{\mu_{j}^{0 P} \bar{\mu}_{i}^{P Q} \mu_{k}^{Q 0}}{\left(E_{0 P}+E_{1}\right)\left(E_{0 Q}-E_{2}\right)}+\right.} \\
& \left.\frac{\mu_{j}^{0 P} \bar{\mu}_{k}^{P Q} \mu_{i}^{Q 0}}{\left(E_{0 P}+E_{1}\right)\left(E_{0 Q}+E_{\xi}\right)}+\frac{\mu_{k}^{0 P} \bar{\mu}_{i}^{P Q} \mu_{j}^{Q 0}}{\left(E_{0 P}+E_{2}\right)\left(E_{0 Q}-E_{1}\right)}+\frac{\mu_{k}^{0 P} \bar{\mu}_{j}^{P Q} \mu_{i}^{Q 0}}{\left(E_{0 P}+E_{2}\right)\left(E_{0 Q}+E_{\xi}\right)}\right] .
\end{aligned}
$$

In the next step, the expanded form of $\beta_{i j k}$ (Eq. 7) is placed in $\beta_{i}$ (Eq. 4). The different components appearing in Eq. 4, i.e., $\beta_{i j j}, \beta_{j i j}$ and $\beta_{j j i}$, are given as

$$
\begin{aligned}
& \beta_{i j j}\left(-\omega_{\xi} ; \omega_{1}, \omega_{2}\right)=\sum_{P, Q}^{\prime}\left[\frac{\mu_{i}^{0 P} \bar{\mu}_{j}^{P Q} \mu_{j}^{Q 0}}{\left(E_{0 P}-E_{\xi}\right)\left(E_{0 Q}-E_{2}\right)}+\frac{\mu_{i}^{0 P} \bar{\mu}_{j}^{P Q} \mu_{j}^{Q 0}}{\left(E_{0 P}-E_{\xi}\right)\left(E_{0 Q}-E_{1}\right)}+\frac{\mu_{j}^{0 P} \bar{\mu}_{i}^{P Q} \mu_{j}^{Q 0}}{\left(E_{0 P}+E_{1}\right)\left(E_{0 Q}-E_{2}\right)}+\right. \\
& \left.\frac{\mu_{j}^{O P} \bar{\mu}_{j}^{P Q} \mu_{i}^{Q 0}}{\left(E_{0 P}+E_{1}\right)\left(E_{0 Q}+E_{\xi}\right)}+\frac{\mu_{j}^{0 P} \bar{\mu}_{i}^{P Q} \mu_{j}^{Q 0}}{\left(E_{0 P}+E_{2}\right)\left(E_{0 Q}-E_{1}\right)}+\frac{\mu_{j}^{0 P} \bar{\mu}_{j}^{P Q} \mu_{i}^{Q 0}}{\left(E_{0 P}+E_{2}\right)\left(E_{0 Q}+E_{\xi}\right)}\right] \text {, } \\
& \beta_{j i j}\left(-\omega_{\xi} ; \omega_{1}, \omega_{2}\right)=\sum_{P, Q}^{\prime}\left[\frac{\mu_{j}^{0 P} \bar{\mu}_{i}^{P Q} \mu_{j}^{Q 0}}{\left(E_{0 P}-E_{\xi}\right)\left(E_{0 Q}-E_{2}\right)}+\frac{\mu_{j}^{0 P} \bar{\mu}_{j}^{P Q} \mu_{i}^{Q 0}}{\left(E_{0 P}-E_{\xi}\right)\left(E_{0 Q}-E_{1}\right)}+\frac{\mu_{i}^{0 P} \bar{\mu}_{j}^{P Q} \mu_{j}^{Q 0}}{\left(E_{0 P}+E_{1}\right)\left(E_{0 Q}-E_{2}\right)}+\right. \\
& \left.\frac{\mu_{i}^{O P} \bar{\mu}_{j}^{P Q} \mu_{j}^{Q 0}}{\left(E_{0 P}+E_{1}\right)\left(E_{0 Q}+E_{\xi}\right)}+\frac{\mu_{j}^{O P} \bar{\mu}_{j}^{P Q} \mu_{i}^{Q 0}}{\left(E_{0 P}+E_{2}\right)\left(E_{0 Q}-E_{1}\right)}+\frac{\mu_{j}^{O P} \bar{\mu}_{i}^{P Q} \mu_{j}^{Q 0}}{\left(E_{0 P}+E_{2}\right)\left(E_{0 Q}+E_{\xi}\right)}\right] \text {, } \\
& \beta_{j j i}\left(-\omega_{\xi} ; \omega_{1}, \omega_{2}\right)=\sum_{P, Q}^{\prime}\left[\frac{\mu_{j}^{0 P} \bar{\mu}_{j}^{P Q} \mu_{i}^{Q 0}}{\left(E_{0 P}-E_{\xi}\right)\left(E_{0 Q}-E_{2}\right)}+\frac{\mu_{j}^{0 P} \bar{\mu}_{i}^{P Q} \mu_{j}^{Q 0}}{\left(E_{0 P}-E_{\xi}\right)\left(E_{0 Q}-E_{1}\right)}+\frac{\mu_{j}^{0 P} \bar{\mu}_{j}^{P Q} \mu_{i}^{Q 0}}{\left(E_{0 P}+E_{1}\right)\left(E_{0 Q}-E_{2}\right)}+\right. \\
& \left.\frac{\mu_{j}^{0 P} \bar{\mu}_{i}^{P Q} \mu_{j}^{Q 0}}{\left(E_{0 P}+E_{1}\right)\left(E_{0 Q}+E_{\xi}\right)}+\frac{\mu_{i}^{0 P} \bar{\mu}_{j}^{P Q} \mu_{j}^{Q 0}}{\left(E_{0 P}+E_{2}\right)\left(E_{0 Q}-E_{1}\right)}+\frac{\mu_{i}^{0 P} \bar{\mu}_{j}^{P Q} \mu_{j}^{Q 0}}{\left(E_{0 P}+E_{2}\right)\left(E_{0 Q}+E_{\xi}\right)}\right] .
\end{aligned}
$$

Adding these three components, we get

$$
\beta_{i j j}+\beta_{j i j}+\beta_{j j i}=\sum_{P, Q}^{\prime} \frac{1}{E_{P Q}}\left[\mu_{i}^{0 P} \bar{\mu}_{j}^{P Q} \mu_{j}^{Q 0}+\mu_{j}^{0 P} \bar{\mu}_{i}^{P Q} \mu_{j}^{Q 0}+\mu_{j}^{0 P} \bar{\mu}_{j}^{P Q} \mu_{i}^{Q 0}\right],
$$

where

$$
\begin{aligned}
\frac{1}{E_{P Q}}= & \frac{1}{\left(E_{0 P}-E_{\xi}\right)\left(E_{0 Q}-E_{2}\right)}+\frac{1}{\left(E_{0 P}-E_{\xi}\right)\left(E_{0 Q}-E_{1}\right)}+\frac{1}{\left(E_{0 P}+E_{1}\right)\left(E_{0 Q}-E_{2}\right)}+\frac{1}{\left(E_{0 P}+E_{1}\right)\left(E_{0 Q}+E_{\xi}\right)}+ \\
& \frac{1}{\left(E_{0 P}+E_{2}\right)\left(E_{0 Q}-E_{1}\right)}+\frac{1}{\left(E_{0 P}+E_{2}\right)\left(E_{0 Q}+E_{\xi}\right)} .
\end{aligned}
$$

We can now use Eqs. 9 and 10 in the different expressions for $\beta$ to identify the contributions from dipole alignment.

\section{A. Dipole alignment in $\beta_{\text {tot }}$}

To extract the dipole alignment contribution to $\beta_{\text {tot }}$, we write $\beta_{x}, \beta_{y}$ and $\beta_{z}$ explicitly and put the square of these quantities in Eq. 3. After arranging the terms in the form of $\mathrm{z}$ scalar product of two vectors, we get

$$
\begin{aligned}
& \beta_{i}= \frac{1}{5} \sum_{P, Q}^{\prime} \frac{1}{E_{P Q}}\left[\mu_{i}^{0 P} \bar{\mu}_{x}^{P Q} \mu_{x}^{Q 0}+\mu_{x}^{O P} \bar{\mu}_{i}^{P Q} \mu_{x}^{Q 0}+\mu_{x}^{O P} \bar{\mu}_{x}^{P Q} \mu_{i}^{Q 0}+\mu_{i}^{0 P} \bar{\mu}_{y}^{P Q} \mu_{y}^{Q 0}+\mu_{y}^{O P} \bar{\mu}_{i}^{P Q} \mu_{y}^{Q 0}+\mu_{y}^{O P} \bar{\mu}_{y}^{P Q} \mu_{i}^{Q 0}+\right. \\
&\left.\mu_{i}^{O P} \bar{\mu}_{z}^{P Q} \mu_{z}^{Q 0}+\mu_{z}^{0 P} \bar{\mu}_{i}^{P Q} \mu_{z}^{Q 0}+\mu_{z}^{O P} \bar{\mu}_{z}^{P Q} \mu_{i}^{Q 0}\right] \\
&=\frac{1}{5} \sum_{P, Q}^{\prime} \frac{1}{E_{P Q}}\left[\mu_{i}^{0 P}\left(\vec{\mu}^{P Q} \cdot \vec{\mu}^{Q 0}\right)+\bar{\mu}_{i}^{P Q}\left(\vec{\mu}^{O P} \cdot \vec{\mu}^{Q 0}\right)+\mu_{i}^{Q 0}\left(\vec{\mu}^{0 P} \cdot \vec{\mu}^{P Q}\right)\right],
\end{aligned}
$$

and

$$
\begin{aligned}
\beta_{i}^{2}=\frac{1}{25_{P, Q, R, S}} \sum^{\prime} \frac{1}{E_{P Q} E_{R S}} & {\left[\mu_{i}^{O P}\left(\vec{\mu}^{P Q} \cdot \vec{\mu}^{Q 0}\right)+\bar{\mu}_{i}^{P Q}\left(\vec{\mu}^{O P} \cdot \vec{\mu}^{Q 0}\right)+\mu_{i}^{Q 0}\left(\vec{\mu}^{O P} \cdot \vec{\mu}^{P Q}\right)\right] } \\
& \times\left[\mu_{i}^{0 R}\left(\vec{\mu}^{R S} \cdot \vec{\mu}^{S 0}\right)+\bar{\mu}_{i}^{R S}\left(\vec{\mu}^{O R} \cdot \vec{\mu}^{S 0}\right)+\mu_{i}^{S 0}\left(\vec{\mu}^{0 R} \cdot \vec{\mu}^{R S}\right)\right] .
\end{aligned}
$$


In Eq. 12, the prime over the summations indicates that none of the four indices $(P, Q, R$, or $S)$ can be the ground state. Inserting Eq. 12 in Eq. 3, we get

$$
\begin{aligned}
& \beta_{\mathrm{tot}}=\sqrt{\beta_{x}^{2}+\beta_{y}^{2}+\beta_{z}^{2}} \\
& =\frac{1}{5}\left(\sum_{P, Q, R, S}^{\prime} \frac{1}{E_{P Q} E_{R S}}\left(\vec{\mu}^{0 P} \cdot \vec{\mu}^{0 R}\right)\left(\vec{\mu}^{Q 0} \cdot \overrightarrow{\vec{\mu}}^{P Q}\right)\left(\vec{\mu}^{S 0} \cdot \overrightarrow{\vec{\mu}}^{R S}\right)+\left(\vec{\mu}^{O P} \cdot \overrightarrow{\vec{\mu}}^{R S}\right)\left(\vec{\mu}^{Q 0} \cdot \overrightarrow{\vec{\mu}}^{P Q}\right)\left(\vec{\mu}^{0 R} \cdot \vec{\mu}^{S 0}\right)+\right. \\
& \left(\vec{\mu}^{O P} \cdot \vec{\mu}^{S 0}\right)\left(\vec{\mu}^{Q 0} \cdot \overrightarrow{\vec{\mu}}^{P Q}\right)\left(\vec{\mu}^{0 R} \cdot \overrightarrow{\vec{\mu}}^{R S}\right)+\left(\vec{\mu}^{0 R} \cdot \overrightarrow{\vec{\mu}}^{P Q}\right)\left(\vec{\mu}^{O P} \cdot \vec{\mu}^{Q 0}\right)\left(\vec{\mu}^{S 0} \cdot \overrightarrow{\vec{\mu}}^{R S}\right)+\left(\overrightarrow{\vec{\mu}}^{R S} \cdot \overrightarrow{\vec{\mu}}^{P Q}\right)\left(\vec{\mu}^{O P} \cdot \vec{\mu}^{Q 0}\right)\left(\vec{\mu}^{O R} \cdot \vec{\mu}^{S 0}\right)+ \\
& \left(\vec{\mu}^{S 0} \cdot \overrightarrow{\vec{\mu}}^{P Q}\right)\left(\vec{\mu}^{O P} \cdot \vec{\mu}^{Q 0}\right)\left(\vec{\mu}^{0 R} \cdot \overrightarrow{\vec{\mu}}^{R S}\right)+\left(\vec{\mu}^{Q 0} \cdot \vec{\mu}^{O R}\right)\left(\vec{\mu}^{O P} \cdot \overrightarrow{\vec{\mu}}^{P Q}\right)\left(\vec{\mu}^{S 0} \cdot \overrightarrow{\vec{\mu}}^{R S}\right)+\left(\vec{\mu}^{Q 0} \cdot \overrightarrow{\vec{\mu}}^{R S}\right)\left(\vec{\mu}^{0 P} \cdot \overrightarrow{\vec{\mu}}^{P Q}\right)\left(\vec{\mu}^{0 R} \cdot \vec{\mu}^{S 0}\right)+ \\
& \left.\left(\vec{\mu}^{Q 0} \cdot \vec{\mu}^{S 0}\right)\left(\vec{\mu}^{O P} \cdot \overrightarrow{\vec{\mu}}^{P Q}\right)\left(\vec{\mu}^{O R} \cdot \overrightarrow{\vec{\mu}}^{R S}\right)\right)^{1 / 2} .
\end{aligned}
$$

Eq.13 can be rewritten as a dipole alignment expression by separating the dot products of the TDMVs into magnitudes and angles as

$$
\begin{aligned}
\beta_{\mathrm{tot}}= & \frac{1}{5}\left(\sum _ { P , Q , R , S } ^ { \prime } \frac { \mu ^ { 0 P } \overline { \mu } ^ { P Q } \mu ^ { Q 0 } \mu ^ { 0 R } \overline { \mu } ^ { R S } \mu ^ { S 0 } } { E _ { P Q } E _ { R S } } \left\{\cos \theta_{0 P}^{0 R} \cos \theta_{0 Q}^{P Q} \cos \theta_{0 S}^{R S}+\cos \theta_{0 P}^{R S} \cos \theta_{0 Q}^{P Q} \cos \theta_{0 R}^{O S}+\cos \theta_{0 P}^{0 S} \cos \theta_{0 Q}^{P Q} \cos \theta_{0 R}^{R S}+\right.\right. \\
& \cos \theta_{0 R}^{P Q} \cos \theta_{0 P}^{0 Q} \cos \theta_{0 S}^{R S}+\cos \theta_{R S}^{P Q} \cos \theta_{0 P}^{0 Q} \cos \theta_{0 R}^{0 S}+\cos \theta_{0 S}^{P Q} \cos \theta_{0 P}^{0 Q} \cos \theta_{0 R}^{R S}+\cos \theta_{0 Q}^{O R} \cos \theta_{0 P}^{P Q} \cos \theta_{0 S}^{R S}+ \\
& \left.\left.\cos \theta_{0 Q}^{R S} \cos \theta_{0 P}^{P Q} \cos \theta_{0 R}^{O S}+\cos \theta_{0 Q}^{0 S} \cos \theta_{0 P}^{P Q} \cos \theta_{0 R}^{R S}\right\}\right)^{1 / 2}
\end{aligned}
$$

where $\mu^{A B}$ represents the magnitude of the TDMV $\vec{\mu}^{A B}$ and $\theta_{A B}^{C D}$ represents the angle between the TDMVs $\vec{\mu}^{A B}$ and $\vec{\mu}^{C D}$. $\beta_{\text {tot }}$ can be written in terms of its elements $\beta_{\text {tot }}^{P Q R S}$ as

$$
\beta_{\mathrm{tot}}=\frac{1}{5} \sqrt{\sum_{P, Q, R, S}^{\prime} \beta_{\mathrm{tot}}^{P Q R S}}
$$

where $\beta_{\mathrm{tot}}^{P Q R S}$ represents everything within the summations in Eq. 14. Thus, $\beta_{\mathrm{tot}}$ can be considered as the sum of $n^{4}$ number of different $\beta_{\mathrm{tot}}^{P Q R S}$ terms, where $n$ is the number of excited states considered in the calculations. For an $m$-state system, $n=m-1$ (as one of the states would be the ground state), there would be $(m-1)^{4}$ number of $\beta_{\mathrm{tot}}^{P Q R S}$ elements.

The expression in curly brackets (angle term) in Eq. 14 is the contribution from the relative orientations of different TDMVs on $\beta_{\mathrm{tot}}$. The angle term of $\beta_{\mathrm{tot}}$ can be positive or negative depending on the alignment of the TDMVs. The maximum value of the angle term (+9) is obtained when the TDMVs $\vec{\mu}^{0 P}, \vec{\mu}^{0 Q}, \overrightarrow{\vec{\mu}} P Q, \vec{\mu}^{O R}, \vec{\mu}^{O S}$ and $\overrightarrow{\vec{\mu}}^{R S}$ are all aligned parallel or when two of them are aligned parallel to each other and anti-parallel to the four remaining TDMVs. An example of the latter is when $\vec{\mu}^{0 Q}$ and $\vec{\mu}^{0 S}$ are aligned anti-parallel to $\vec{\mu}^{0 P}, \overrightarrow{\vec{\mu}}^{P Q}, \vec{\mu}^{O R}$ and $\overrightarrow{\vec{\mu}}^{R S}$. The minimum value of the angle term (-9) is obtained when one or three TDMVs are aligned anti-parallelly to all others, such as when $\vec{\mu}^{O P}, \vec{\mu}^{0 Q}$ and $\overrightarrow{\vec{\mu}} P Q$ are parallel to each other and anti-parallel to $\vec{\mu}^{0 R}, \vec{\mu}^{0 S}$ and $\overrightarrow{\vec{\mu}}^{R S}$.

Comparing Eq. 14 with the generalized few-state model formula ${ }^{35,37}$ for two-photon absorption (2PA) or three-photon absorption (3PA), it is clear that at a molecular level, the expression for $\beta$ is much more complicated than the corresponding expressions for a multiphoton absorption process. Since there is no final state in the case of $\beta$, the number of dipoles involved is much larger and hence also the number of orientation terms.

If the two transition channels $0 \rightarrow P \rightarrow Q \rightarrow 0$ and $0 \rightarrow R \rightarrow S \rightarrow 0$ are the same $(P=R$ and $Q=S)$, the expression for $\beta_{\text {tot }}$ is considerably simplified:

$$
\beta_{\mathrm{tot}}^{P=R, Q=S}=\frac{1}{5} \sum_{P, Q}^{\prime} \frac{\mu^{0 P} \bar{\mu}^{P Q} \mu^{Q 0}}{E_{P Q}} \sqrt{6 \cos \theta_{0 P}^{0 Q} \cos \theta_{0 P}^{P Q} \cos \theta_{0 Q}^{P Q}+\cos ^{2} \theta_{0 P}^{0 Q}+\cos ^{2} \theta_{0 P}^{P Q}+\cos ^{2} \theta_{0 Q}^{P Q}} .
$$

\section{B. Dipole alignment in $\beta_{\|}$}

In order to derive the dipole alignment formula for $\beta_{\|}$, we insert Eq. 9 into Eq. 6a. This gives

$$
\beta_{\|}=\frac{1}{5} \sum_{i, j} \sum_{P, Q}^{\prime} \frac{1}{E_{P Q}}\left[\mu_{i}^{0 P} \bar{\mu}_{j}^{P Q} \mu_{j}^{Q 0}+\mu_{j}^{0 P} \bar{\mu}_{i}^{P Q} \mu_{j}^{Q 0}+\mu_{j}^{O P} \bar{\mu}_{j}^{P Q} \mu_{i}^{Q 0}\right] \frac{\mu_{i}^{00}}{\mu^{00}} .
$$


On expanding over the Cartesian coordinates (i.e., over the indices $i$ and $j$ ), we get

$$
\begin{aligned}
\mu^{00} \beta_{\|}= & \frac{1}{5} \sum_{P, Q}^{\prime} \frac{1}{E_{P Q}}\left[3 \mu_{x}^{0 P} \bar{\mu}_{x}^{P Q} \mu_{x}^{Q 0} \mu_{x}^{00}+\mu_{x}^{0 P} \bar{\mu}_{y}^{P Q} \mu_{y}^{Q 0} \mu_{x}^{00}+\mu_{y}^{0 P} \bar{\mu}_{x}^{P Q} \mu_{y}^{Q 0} \mu_{x}^{00}+\mu_{y}^{0 P} \bar{\mu}_{y}^{P Q} \mu_{x}^{Q 0} \mu_{x}^{00}+\mu_{x}^{0 P} \bar{\mu}_{z}^{P Q} \mu_{z}^{Q 0} \mu_{x}^{00}+\right. \\
& \mu_{z}^{0 P} \bar{\mu}_{x}^{P Q} \mu_{z}^{Q 0} \mu_{x}^{00}+\mu_{z}^{0 P} \bar{\mu}_{z}^{P Q} \mu_{x}^{Q 0} \mu_{x}^{00}+\mu_{y}^{0 P} \bar{\mu}_{x}^{P Q} \mu_{x}^{Q 0} \mu_{y}^{00}+\mu_{x}^{0 P} \bar{\mu}_{y}^{P Q} \mu_{x}^{Q 0} \mu_{y}^{00}+\mu_{x}^{0 P} \bar{\mu}_{x}^{P Q} \mu_{y}^{Q 0} \mu_{y}^{00}+3 \mu_{y}^{0 P} \bar{\mu}_{y}^{P Q} \mu_{y}^{Q 0} \mu_{y}^{00}+ \\
& \mu_{y}^{0 P} \bar{\mu}_{z}^{P Q} \mu_{z}^{Q 0} \mu_{y}^{00}+\mu_{z}^{0 P} \bar{\mu}_{y}^{P Q} \mu_{z}^{Q 0} \mu_{y}^{00}+\mu_{z}^{0 P} \bar{\mu}_{z}^{P Q} \mu_{y}^{Q 0} \mu_{y}^{00}+\mu_{z}^{0 P} \bar{\mu}_{x}^{P Q} \mu_{x}^{Q 0} \mu_{z}^{00}+\mu_{x}^{0 P} \bar{\mu}_{z}^{P Q} \mu_{x}^{Q 0} \mu_{z}^{00}+\mu_{x}^{0 P} \bar{\mu}_{x}^{P Q} \mu_{z}^{Q 0} \mu_{z}^{00}+ \\
& \left.\mu_{z}^{0 P} \bar{\mu}_{y}^{P Q} \mu_{y}^{Q 0} \mu_{z}^{00}+\mu_{y}^{0 P} \bar{\mu}_{z}^{P Q} \mu_{y}^{Q 0} \mu_{z}^{00}+\mu_{y}^{0 P} \bar{\mu}_{y}^{P Q} \mu_{z}^{Q 0} \mu_{z}^{00}+3 \mu_{z}^{0 P} \bar{\mu}_{z}^{P Q} \mu_{z}^{Q 0} \mu_{z}^{00}\right] \\
= & \frac{1}{5} \sum_{P, Q}^{\prime}\left[\left(\vec{\mu}^{0 P} \cdot \vec{\mu}^{00}\right)\left(\vec{\mu}^{P Q} \cdot \vec{\mu}^{Q 0}\right)+\left(\vec{\mu}^{0 P} \cdot \overrightarrow{\vec{\mu}}^{P Q}\right)\left(\overrightarrow{\bar{\mu}}^{Q 0} \cdot \vec{\mu}^{00}\right)+\left(\vec{\mu}^{0 P} \cdot \vec{\mu}^{Q 0}\right)\left(\overrightarrow{\vec{\mu}}^{P Q} \cdot \vec{\mu}^{00}\right)\right] .
\end{aligned}
$$

Eq. 18 can be rewritten as a dipole alignment expression by separating the dot products of the TDMVs into magnitudes and angles as

$$
\beta_{\|}=\frac{1}{5} \sum_{P, Q}^{\prime} \frac{\mu^{0 P} \bar{\mu}^{P Q} \mu^{Q 0}}{E_{P Q}}\left\{\cos \theta_{0 P}^{00} \cos \theta_{P Q}^{0 Q}+\cos \theta_{0 P}^{P Q} \cos \theta_{0 Q}^{00}+\cos \theta_{0 P}^{0 Q} \cos \theta_{P Q}^{00}\right\},
$$

or in terms of its components $\beta_{\|}^{P Q}$ as

$$
\beta_{\|}=\frac{1}{5} \sum_{P, Q}^{\prime} \beta_{\|}^{P Q}
$$

The angle term of $\beta_{\|}$(the term in curly brackets in Eq. 19) can be positive or negative depending on the alignment of the TDMVs. The maximum value of the angle term (+3) is obtained when the TDMVs $\vec{\mu}^{00}, \vec{\mu}^{0 P}, \vec{\mu}^{0 Q}$ and $\mu^{\vec{P} Q}$ are all aligned parallel or when two of them are aligned parallel to each other and anti-parallel to the two remaining TDMVs. The minimum value of the angle term $(-3)$ is obtained when one of the four TDMVs involved is anti-parallel to the other three, such as when $\vec{\mu}^{00}$ is anti-parallel to $\vec{\mu}^{O P}, \vec{\mu}^{0 Q}$ and $\overrightarrow{\vec{\mu}}^{P Q}$.

$\beta_{\|}$equals the sum of $n^{2}$ number of $\beta_{\|}^{P Q}$ terms, where $n$ is the number of excited states in the system. Eq. 19 clearly indicates that the expression for the contribution of dipole alignment on $\beta_{\|}$is much simpler than the corresponding expression for $\beta_{\text {tot }}$ (Eq. 14). Indeed, $\beta_{\|}$is only the vector component of $\beta$ along the ground-state dipole moment. Each term in Eq. 19 involves at most three different TDMVs, which is half as many as appearing in Eq. 14. The magnitude of the ground-state dipole moment does not appear in Eq. 19, but its direction is needed to evaluate the angle term.

\section{Dipole alignment in $\beta_{\perp}$}

Similar to the case for $\beta_{\|}$, the dipole alignment formula of $\beta_{\perp}$ can be derived by inserting Eqs. $8 \mathrm{a}, 8 \mathrm{~b}$ and $8 \mathrm{c}$ into Eq. $6 \mathrm{~b}$. A closer inspection of the resulting expressions reveals that the summation $\left(2 \beta_{i j j}-3 \beta_{j i j}+2 \beta_{j j i}\right)$ gives an equation similar to Eq. 9:

$$
2 \beta_{i j j}-3 \beta_{j i j}+2 \beta_{j j i}=\sum_{P, Q}^{\prime} \frac{1}{E_{P Q}^{\prime}}\left[\mu_{i}^{0 P} \bar{\mu}_{j}^{P Q} \mu_{j}^{Q 0}+\mu_{j}^{0 P} \bar{\mu}_{i}^{P Q} \mu_{j}^{Q 0}+\mu_{j}^{0 P} \bar{\mu}_{j}^{P Q} \mu_{i}^{Q 0}\right],
$$

where $E_{P Q}^{\prime}$ is given as

$$
\begin{aligned}
\frac{1}{E_{P Q}^{\prime}}= & \frac{2}{\left(E_{0 P}-E_{\xi}\right)\left(E_{0 Q}-E_{2}\right)}+\frac{2}{\left(E_{0 P}-E_{\xi}\right)\left(E_{0 Q}-E_{1}\right)}-\frac{3}{\left(E_{0 P}+E_{1}\right)\left(E_{0 Q}-E_{2}\right)}-\frac{3}{\left(E_{0 P}+E_{1}\right)\left(E_{0 Q}+E_{\xi}\right)}+ \\
& \frac{2}{\left(E_{0 P}+E_{2}\right)\left(E_{0 Q}-E_{1}\right)}+\frac{2}{\left(E_{0 P}+E_{2}\right)\left(E_{0 Q}+E_{\xi}\right)} .
\end{aligned}
$$

The dipole alignment expression for $\beta_{\perp}$ is thus given as

$$
\beta_{\perp}=\frac{1}{5} \sum_{P, Q}^{\prime} \frac{\mu^{0 P} \bar{\mu}^{P Q} \mu^{Q 0}}{E_{P Q}^{\prime}}\left\{\cos \theta_{0 P}^{00} \cos \theta_{P Q}^{0 Q}+\cos \theta_{0 P}^{P Q} \cos \theta_{0 Q}^{00}+\cos \theta_{0 P}^{0 Q} \cos \theta_{P Q}^{00}\right\},
$$


or in terms of its components $\beta_{\perp}^{P Q}$ as

$$
\beta_{\perp}=\frac{1}{5} \sum_{P, Q}^{\prime} \beta_{\perp}^{P Q}
$$

The only difference between the dipole alignment expressions for $\beta_{\|}$(Eq. 19) and $\beta_{\perp}$ (Eq. 23) is the energy term, which is given by Eq. 10 for $\beta_{\|}$and by Eq. 22 for $\beta_{\perp}$. Thus, the angle term (the term in curly brackets in Eq. 23) can assume values between -3 and +3 with the same maximization and minimization conditions as for $\beta_{\|}$.

\section{General expression}

The dipole alignment expressions of $\beta_{\mathrm{tot}}, \beta_{\|}$and $\beta_{\perp}$ can be written in a general form as

$$
\beta=\frac{1}{5}\left(\Sigma^{\prime} \frac{\text { dipole term }}{\text { energy term }} \times \text { angle term }\right)^{y},
$$

where the exponent $y$ is $1 / 2$ for $\beta_{\text {tot }}$ and 1 for $\beta_{\|}$and $\beta_{\perp}$. The summation is over four indices for $\beta_{\text {tot }}$ and over two indices for $\beta_{\|}$and $\beta_{\perp}$. The dipole term is a product of the magnitude of the six TDMVs involved in $\beta_{\mathrm{tot}}^{P Q R S}$ and the three TDMVs involved in $\beta_{\|}^{P Q}$ and $\beta_{\perp}^{P Q}$. Hence, the dipole term is always positive. The dipole term in $\beta_{\text {tot }}$ represents two transition channels viz. $0 \rightarrow P \rightarrow Q \rightarrow 0$ and $0 \rightarrow R \rightarrow S \rightarrow 0$ because of the involvement of quadratic terms such as $\beta_{i}^{2}$. The corresponding angle term represents the interference between the two channels. This interpretation is equivalent to the channel interference picture of multiphoton absorption processes. ${ }^{37} \beta_{\|}$ and $\beta_{\perp}$, on the other hand, do not contain any quadratic term and thus the corresponding dipole term represents only one transition channel.

\section{FEW-STATE MODELS BASED ON DIPOLE ALIGNMENT EXPRESSIONS}

The dipole alignment expressions derived in the previous section involve summations over all the excited states in the system. In practical calculations, this summation is not feasible except for some very simple systems. Therefore, one has to truncate the summation in the expressions, giving rise to so-called few-state models. Few-state models for any given number of states can be derived from the expressions in the previous section. We will here derive explicit expressions for few-state models with two and three states.

\section{A. Two-state model}

The simplest of the few-state models is the two-state model (2SM). In a $2 \mathrm{SM}$, the indices of the summation are either the ground state $|0\rangle$ or a particular excited state that we will here denote as $|P\rangle$. Since the summation indices cannot be the ground state in the dipole alignment expressions derived in the previous section, all summation indices take the value of the excited state $|P\rangle$. Therefore, the $2 \mathrm{SM}$ expressions for $\beta_{\mathrm{tot}}, \beta_{\|}$ and $\beta_{\perp}$ are

$$
\begin{aligned}
& \beta_{\mathrm{tot}}^{2 \mathrm{SM}}=\frac{1}{5} \frac{\left(\mu^{0 P}\right)^{2} \bar{\mu}^{P P}}{E_{P P}} \sqrt{8 \cos ^{2} \theta_{0 P}^{P P}+1} \\
& \beta_{\|}^{2 \mathrm{SM}}=\frac{1}{5} \frac{\left(\mu^{0 P}\right)^{2} \bar{\mu}^{P P}}{E_{P P}}\left\{2 \cos \theta_{0 P}^{00} \cos \theta_{0 P}^{P P}+\cos \theta_{00}^{P P}\right\} \\
& \beta_{\perp}^{2 \mathrm{SM}}=\frac{1}{5} \frac{\left(\mu^{0 P}\right)^{2} \bar{\mu}^{P P}}{E_{P P}^{\prime}}\left\{2 \cos \theta_{0 P}^{00} \cos \theta_{0 P}^{P P}+\cos \theta_{00}^{P P}\right\}
\end{aligned}
$$

where $\bar{\mu}_{P P}=\left|\vec{\mu}^{P P}-\vec{\mu}^{00}\right|$. Several interesting observations can be made by evaluating these formulae. First, $\beta_{\mathrm{tot}}^{2 \mathrm{SM}} \mathrm{de}-$ pends on only one angle, namely the angle $\theta_{0 P}^{P P}$ between $\vec{\mu}^{0 P}$ and $\overrightarrow{\vec{\mu}}^{P P}$, whereas $\beta_{\|}^{2 \mathrm{SM}}$ and $\beta_{\perp}^{2 \mathrm{SM}}$ depend on all three possible angles $\theta_{00}^{O P}, \theta_{0 P}^{P P}$ and $\theta_{00}^{P P}$. The angle term in $\beta_{\text {tot }}^{2 \mathrm{SM}}$ is always positive and reaches its maximum value when $\vec{\mu}^{O P}$ and $\overrightarrow{\vec{\mu}}^{P P}$ are parallel or anti-parallel. The angle term in $\beta_{\|}^{2 S M}$ and $\beta_{\perp}^{2 S M}$ can be either positive or negative. The maximum value of +3 is obtained when $\vec{\mu}^{00}, \vec{\mu}^{O P}$ and $\overrightarrow{\vec{\mu}}^{P P}$ are all aligned in parallel or when $\vec{\mu}^{00}$ and $\overrightarrow{\vec{\mu}}^{P P}$ are aligned parallel to each other and anti-parallel to $\vec{\mu}^{O P}$. The minimum value of -3 is obtained when $\vec{\mu}^{00}$ and $\vec{\mu}^{P P}$ are aligned anti-parallel to each other with $\vec{\mu}^{O P}$ parallel to either $\vec{\mu}^{00}$ or $\overrightarrow{\vec{\mu}}^{P P} . \beta_{\text {tot }}^{2 S M}$ and $\beta_{\|}^{2 S M}$ only differ in the angle term. In all maximization and minimization conditions for $\beta_{\|}^{2 S M}, \beta_{\text {tot }}^{2 S M}$ only has a component in the direction of the dipole moment. Thus, $\beta_{\mathrm{tot}}^{2 S M}=\beta_{\|}^{2 S M}$ when the maximization conditions hold and $\beta_{\mathrm{tot}}^{2 S M}=-\beta_{\|}^{2 S M}$ when the minimization conditions hold. Finally, the ratio of isotropically averaged parallel and perpendicular $\beta$, within the 2SM, is equal to the inverse ratio of the corresponding energy terms, i.e.,

$$
\frac{\beta_{\|}^{2 \mathrm{SM}}}{\beta_{\perp}^{2 \mathrm{SM}}}=\frac{E_{P P}^{\prime}}{E_{P P}}
$$

\section{B. Three-state model}

The three-state model (3SM) expressions for $\beta_{\mathrm{tot}}, \beta_{\|}$, and $\beta_{\perp}$ are obtained by considering the ground state and two different excited states in the summations in Eqs. 14, 19 and 23. Calling the excited states $A$ and $B$, i.e., $P, Q, R, S=A, B$, we 
can write

$$
\begin{array}{r}
\beta_{\mathrm{tot}}^{3 \mathrm{SM}}=\frac{1}{5}\left(\beta_{\mathrm{tot}}^{\mathrm{AAAA}}+\beta_{\mathrm{tot}}^{\mathrm{AAAB}}+\beta_{\mathrm{tot}}^{\mathrm{AABA}}+\beta_{\mathrm{tot}}^{\mathrm{AABB}}+\right. \\
\beta_{\mathrm{tot}}^{\mathrm{ABAA}}+\beta_{\mathrm{tot}}^{\mathrm{ABAB}}+\beta_{\mathrm{tot}}^{\mathrm{ABBA}}+\beta_{\mathrm{tot}}^{\mathrm{ABBB}}+ \\
\beta_{\mathrm{tot}}^{\mathrm{BAAA}}+\beta_{\mathrm{tot}}^{\mathrm{BAAB}}+\beta_{\mathrm{tot}}^{\mathrm{BABA}}+\beta_{\mathrm{tot}}^{\mathrm{BABB}}+ \\
\left.\beta_{\mathrm{tot}}^{\mathrm{BBAA}}+\beta_{\mathrm{tot}}^{\mathrm{BBAB}}+\beta_{\mathrm{tot}}^{\mathrm{BBBA}}+\beta_{\mathrm{tot}}^{\mathrm{BBBB}}\right)^{1 / 2}, \\
\beta_{\| \text {or } \perp=}^{3 \mathrm{SM}}=\frac{1}{5}\left(\beta_{\| \text {or } \perp}^{\mathrm{AA}}+\beta_{\| \text {or } \perp}^{\mathrm{AB}}+\beta_{\| \text {or } \perp}^{\mathrm{BA}}+\beta_{\| \text {or } \perp}^{\mathrm{BB}}\right) .
\end{array}
$$

Each term in these equations (Eqs. 30 and 31) can be obtained from the respective expressions in Eqs. 14, 19 and 23. Terms such as $\beta_{\mathrm{tot}}^{\mathrm{AAAA}}$ or $\beta_{\| \mathrm{A} \perp}^{\mathrm{AA}}$, i.e., those having only one type of index, appear also in the 2SM. The expression for the simplest non-2SM term for $\beta_{\mathrm{tot}}^{3 \mathrm{SM}}$ is given below as an example

$$
\begin{aligned}
\beta_{\text {tot }}^{\mathrm{AAAB}}= & \frac{\left(\mu^{0 A}\right)^{3} \mu^{0 B} \bar{\mu}^{A A} \bar{\mu}^{A B}}{E_{A A} E_{A B}} \times\left(3 \cos \theta_{0 \mathrm{~A}}^{\mathrm{AA}} \cos \theta_{0 \mathrm{~B}}^{\mathrm{AB}}\right. \\
& +\cos \theta_{\mathrm{AB}}^{\mathrm{AA}} \cos \theta_{0 \mathrm{~A}}^{0 \mathrm{~B}}+\cos \theta_{0 \mathrm{~B}}^{\mathrm{AA}} \cos \theta_{0 \mathrm{~A}}^{\mathrm{AB}} \\
& \left.+4 \cos \theta_{0 \mathrm{~A}}^{\mathrm{AB}} \cos \theta_{0 \mathrm{~A}}^{\mathrm{AA}} \cos \theta_{0 \mathrm{~A}}^{0 \mathrm{~B}}\right) .
\end{aligned}
$$

In general, $\beta_{\mathrm{tot}}^{\mathrm{AAAB}}=\beta_{\mathrm{tot}}^{\mathrm{AABA}}=\beta_{\mathrm{tot}}^{\mathrm{ABAA}}=\beta_{\mathrm{tot}}^{\mathrm{BAAA}}$. The expressions for $\beta_{\| \text {or } \perp}^{\mathrm{AB}}$ are already given in Eqs. 19 and 23 as $\beta_{\| \text {or } \perp \text {. }}^{P Q}$.

\section{DIPOLE ALIGNMENT EXPRESSIONS FOR THE STATIC $\beta$}

In all the above treatment, we have not mentioned the values of the three frequencies or energies $E_{\xi}, E_{1}$ and $E_{2}$. Therefore, the expressions derived in the previous sections are valid for both static as well as dynamic $\beta$ s. The static case has a much simpler expression for the energy term, which is obtained by putting $E_{\xi}=E_{1}=E_{2}=0$ in Eqs. 10 and 22. Thus,

$$
\frac{1}{E_{P Q}^{\mathrm{s}}}=\frac{6}{E_{0 P} E_{0 Q}} \quad \text { and } \quad \frac{1}{E_{P Q}^{S,}}=\frac{2}{E_{0 P} E_{0 Q}},
$$

where he superscript ' $s$ ' refers to the static case. All the other terms remain unchanged.

Since $\beta_{\|}$(Eq. 19) and $\beta_{\perp}$ (Eq. 23) only differ in the energy term, their static counterparts differ by a factor of three as

$$
\beta_{\|}^{s}=3 \beta_{\perp}^{s} .
$$

Expressions for $\beta^{s}$ in a $2 \mathrm{SM}$ are obtained by inserting Eq. 33 into Eqs. $26-28$ and are given as

$$
\begin{aligned}
& \beta_{\mathrm{tot}}^{s, 2 S M}=\frac{6}{5} \frac{\left(\mu^{O P}\right)^{2} \bar{\mu}^{P P}}{E_{0 P}^{2}} \sqrt{8 \cos ^{2} \theta_{0 P}^{P P}+1}, \\
& \beta_{\|}^{s, 2 S M}=\frac{6}{5} \frac{\left(\mu^{O P}\right)^{2} \bar{\mu}^{P P}}{E_{0 P}^{2}}\left\{2 \cos \theta_{0 P}^{00} \cos \theta_{0 P}^{P P}+\cos \theta_{00}^{P P}\right\}, \\
& \beta_{\perp}^{s, 2 S M}=\frac{2}{5} \frac{\left(\mu^{O P}\right)^{2} \bar{\mu}^{P P}}{E_{0 P}^{2}}\left\{\left(2 \cos \theta_{0 P}^{00} \cos \theta_{0 P}^{P P}+\cos \theta_{00}^{P P}\right\} .\right.
\end{aligned}
$$

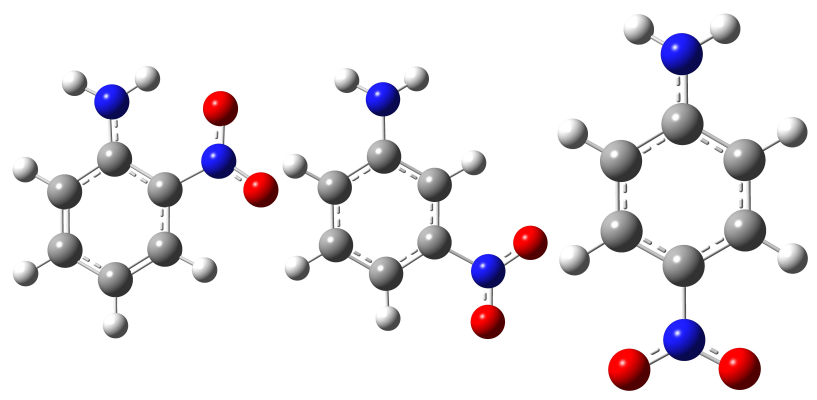

FIG. 1. Vacuum-phase optimized geometries of $o-, m$ - and $p$ nitroaniline

We note that the energy terms in Eq. 33 are always positive for static $\beta \mathrm{s}$. Thus, $\beta_{\mathrm{tot}}^{s, 2 S M}$ is always positive and only the angle term can contribute to a negative component in $\beta_{\mathrm{tot}}^{s, P Q}$, $\beta_{\|}^{s, P Q}$ or $\beta_{\perp}^{s . P Q}$.

\section{ILLUSTRATION OF DIPOLE ALIGNMENT IN $O-, M-$ AND P-NITROANILINE (ONA, MNA, AND PNA)}

We have applied the derived expressions (Eqs. 14, 19 and 23) to investigate the contribution of dipole alignment on static $\beta$ s of $o-, m-$, and $p$-nitroaniline (ONA, MNA, and PNA). The ground-state geometries were optimized at the B3LYP/aug-cc-pVDZ level of theory using Gaussian16. ${ }^{48}$ Optimized geometries are shown in Figure 1.

Excitation energies as well as TDMVs for ground-state to excited-state and excited-state to excited-state transitions were calculated for 50 excited states at the time-dependent density functional theory level of theory ${ }^{49}$ using CAMB3LYP/aug-ccpVDZ as implemented in the LSDalton ${ }^{50,51}$ program package. $\beta_{\text {tot }}, \beta_{\|}$and $\beta_{\perp}$ were calculated with different few-state models using a computer code that has been developed to treat the equations derived in this work. The code is available as open source. ${ }^{52}$ Results for $\beta^{s}$ are shown in Figure 2. It is important to mention here that few-state models can be constructed in different ways, e.g. a four-state model can be considered by including states $0,1,2,3$ or $0,1,2,4$, etc. Here an $n$-state model is constructed by considering all consecutive states from the ground state to excited state $n-1$. Response theory results were calculated at the same level of theory in LSDalton for $\beta_{\mathrm{tot}}, \beta_{\|}$and $\beta_{\perp}$ for reference, and are given in Table I and as horizontal lines in Figure 2.

\begin{tabular}{|c|c|c|c|}
\hline & ONA & MNA & PNA \\
\hline$\beta_{\text {tot }}$ & 281.670 & 296.265 & 834.100 \\
\hline$\beta_{\|}$ & 268.139 & 280.827 & 829.583 \\
\hline$\beta_{\perp}$ & 89.380 & 93.609 & 276.528 \\
\hline
\end{tabular}

TABLE I. Response theory results for $\beta_{\mathrm{tot}}, \beta_{\|}$, and $\beta_{\perp}$ for ONA, MNA, and PNA.

The few-state model results converge reasonably well to the response theory results: to within $25 \%$ for ONA and MNA and to within $2.5 \%$ for PNA with 50 excited states. After the 

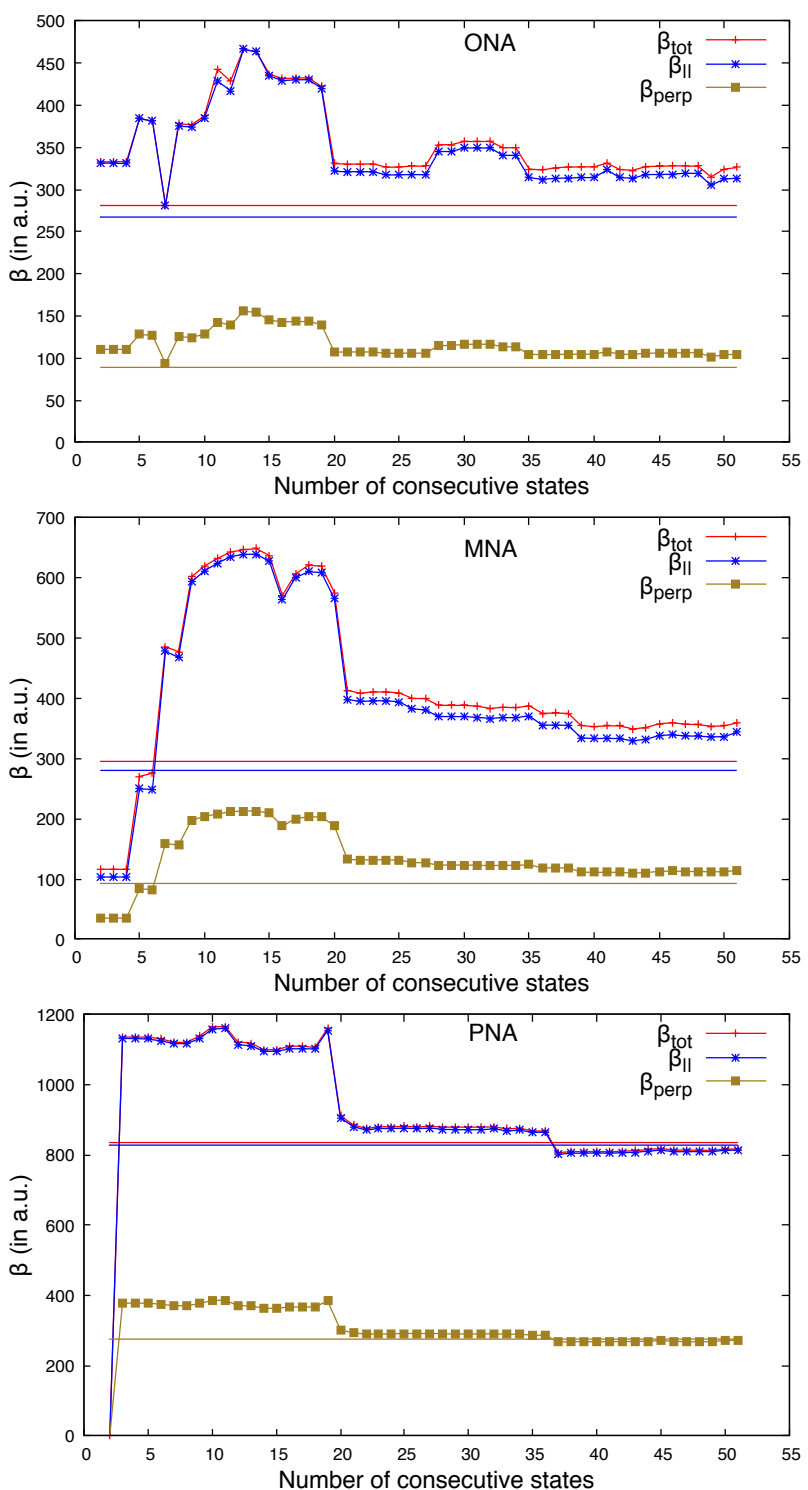

FIG. 2. $\beta_{\mathrm{tot}}^{s}, \beta_{\|}^{s}$ and $\beta_{\perp}^{s}\left(\beta_{\mathrm{perp}}\right)$ for ONA, MNA and PNA calculated with $n$-state models from $n=2$ to $n=50$. The response theory results are shown as horizontal lines.

$20^{\text {th }}$ state, no single excited state has a large impact on the SOS results. Contributions to $\beta^{s}$ can be positive or negative. Since the dipole term and energy term are always positive for $\beta^{s}$, it is the sign of the angle term that determines whether the contribution to $\beta^{s}$ is positive or negative.

Interestingly, the values of $\beta_{\mathrm{tot}}^{s}$ and $\beta_{\|}^{s}$ are similar for ONA and MNA and almost the same for PNA. $\beta_{\perp}^{s}$ is exactly one third of $\beta_{\|}^{s}$ as is always the case for $\beta^{s}$ (Eq. 34). In order to demonstrate the importance of dipole alignment, we have also computed few-state model results assuming that all TDMVs are parallel to each other, i.e., assuming that each cosine in the angle term is 1 . The results are plotted in Figure 3. The angle term is in this case 9 for $\beta_{\text {tot }}^{s}$ (Eq. 14) and 3 for $\beta_{\|}^{s}$ and $\beta_{\perp}^{s}$ (Eqs. 19 and 23). The results in Figure 3 show no sign
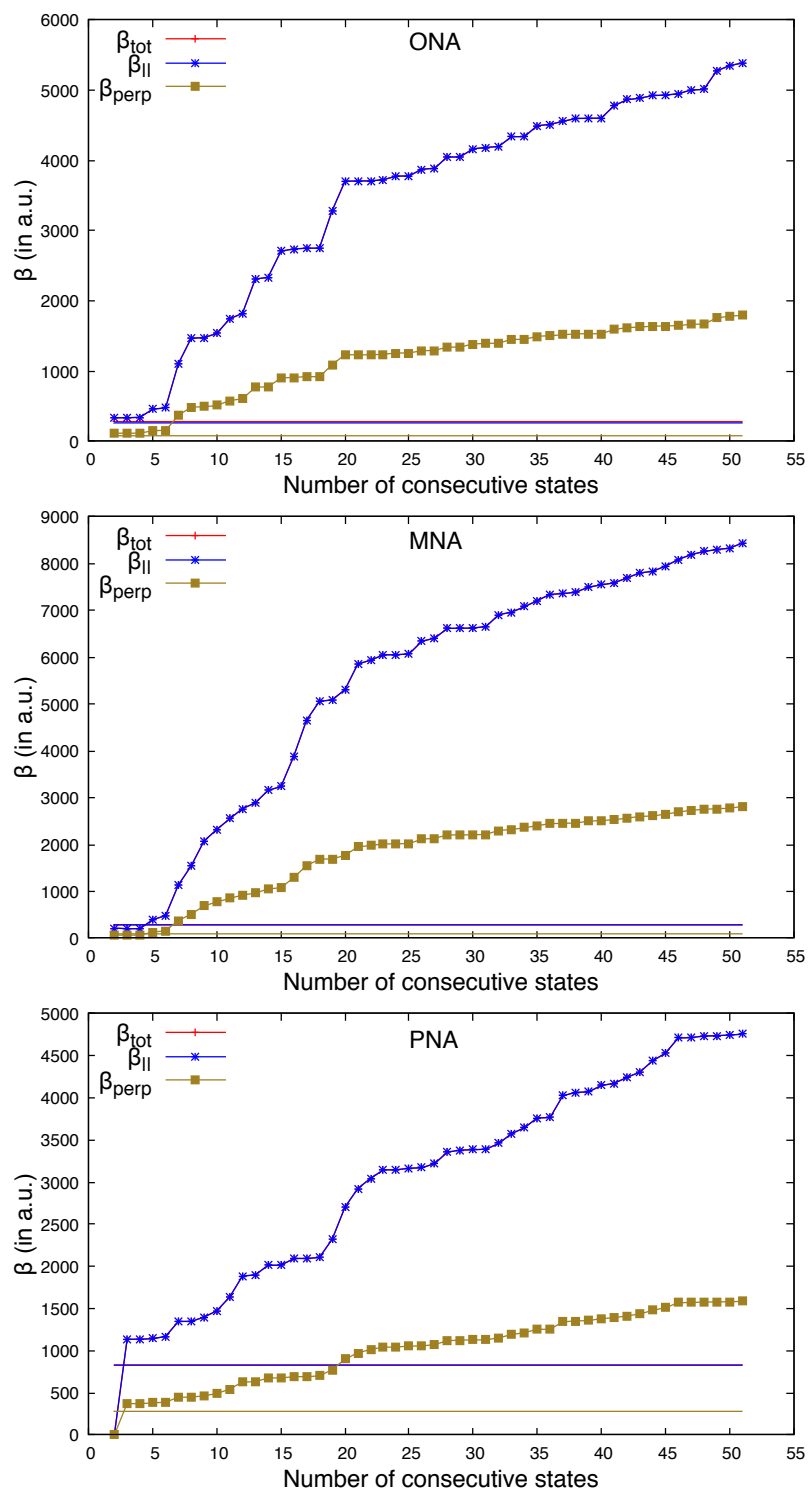

FIG. 3. Convergence of $\beta_{\mathrm{tot}}, \beta_{\|}$, and $\beta_{\perp}\left(\beta_{\mathrm{perp}}\right)$ with few-state models in ONA, MNA and PNA, when each cosine in the angle term is assumed to be 1.0. In this particular case, $\beta_{\mathrm{tot}}=\beta_{\|}$. The response theory results are shown as horizontal lines.

of convergence and the values are significantly overestimated compared to the response value results. Indeed, convergence is not possible because all contributions to $\beta^{s}$ are positive. Large negative contributions to $\beta^{s}$ such as when including state number 20 (Figure 2) are large positive contributions in Figure 3. This clearly shows that it is important to include the effect of dipole alignment in the SOS expression of $\beta$. Note that the values of $\beta_{\mathrm{tot}}$ and $\beta_{\|}$are the same when the two transition channels in $\beta_{\text {tot }}$ do not interact through the angle term. The component $\beta_{\mathrm{tot}}^{P Q R S}$ reduces to the square of the component $\beta_{\|}^{P Q}$ giving equivalent values for $\beta_{\mathrm{tot}}$ (Eq. 15) and $\beta_{\|}$(Eq. 20). 


\section{CONCLUSIONS}

Non-linear optical properties such as multiphoton absorption and $\beta$ involve more than one TDMV and hence these properties depend not only on the magnitude but also on the relative orientation of these vectors. In this work, we have derived the generalized few-state model formulae for quantifying the importance of the relative orientation of different TMDVs on $\beta \mathrm{s}-\beta_{\mathrm{tot}}$ and the isotropically averaged parallel $\left(\beta_{\|}\right)$and perpendicular $\left(\beta_{\perp}\right)$-in line with out previous work on multiphoton absorption. Our derivations are general in the sense that they can be used to generate any few-state model formula and applied to frequency-dependent as well as static $\beta \mathrm{s}$. We have applied the derived formulae to illustrate the importance of relative orientations of different TDMVs on $\beta \mathrm{s}$ in $o-, m$-, and $p$-nitroaniline. This work opens new ways to understand $\beta$ and allows to establish structure-property relationship between the TDMV orientations and different $\beta \mathrm{s}$. An extension of the generalized few-state model of $\beta$ to coupledcluster theory will be published in a forthcoming work.

\section{SUPPLEMENTARY MATERIAL}

The optimized Cartesian coordinates for the ground state geometries of all the three systems considered in this work and the data required to reproduce the plots in this manuscript are provided as supplementary material.

\section{ACKNOWLEDGMENTS}

The authors gratefully acknowledge support from the Norwegian Supercomputing facility (NOTUR, Grant No. NN4654K) and from the Research Council of Norway through a Centre of Excellence grant (Grant No. 262695) and a research grant (Grant No. 250743). MMA acknowledges the "Research Initiation Grant (IIT Bhilai/D/2258)" provided by the Indian Institute of Technology Bhilai.

\section{DATA AVAILABILITY STATEMENT}

The data that supports the findings of this study are available within the article [and its supplementary material].

${ }^{1}$ C. D. Andrade, C. O. Yanez, L. Rodriguez, and K. D. Belfield, "A series of fluorene-based two-photon absorbing molecules: Synthesis, linear and nonlinear characterization, and bioimaging," J. Org. Chem. 75, 3975-3982 (2010).

${ }^{2}$ S. Brown, "Two photons are better than one," Nat. Photon 2, 394-395 (2008).

${ }^{3}$ R. R. Avirah, D. T. Jayaram, N. Adarsh, and D. Ramaiah, "Squaraine dyes in pdt: from basic design to in vivo demonstration," Org. Biomol. Chem. 10, 911-920 (2012)

${ }^{4}$ P. Steglich, C. Villringer, B. Dietzel, C. Mai, S. Schrader, M. Casalboni, and A. Mai, "On-chip dispersion measurement of the quadratic electrooptic effect in nonlinear optical polymers using a photonic integrated circuit technology," IEEE Photon. J. 11, 1-10 (2019).
${ }^{5}$ B. H. Cumpston, S. P. Ananthavel, S. Barlow, D. L. Dyer, jeffrey E. Ehrlich, L. L. Erskine, A. A. Heikal, S. M. Kuebler, I.-Y. S. Lee, D. McCordMaughon, J. Qin, H. Röckel, M. Rumi, X.-L. Wu, S. R. Marder, and J. W. Perry, "Two-photon polymerization initiators for three-dimensional optical data storage and microfabrication." Nature 398, 51-54 (1999).

${ }^{6}$ R. L. Sutherland, Handbook of nonlinear optics (Marcel Dekker, Inc., 2003).

${ }^{7}$ R. Boyd, Nonlinear optics (Elsevier, 2008)

${ }^{8} \mathrm{~K}$. Kikuchi and K. Tada, "Theory of electric field-induced optical second harmonic generation in semiconductors," Opt. Quant. Electron. 12, 199205 (1980).

${ }^{9} \mathrm{~F}$. Kajzar, "Electric-field-induced second harmonic generation" in Materials Characterization and Optical Probe Techniques: A Critical Review, Vol. 10291, edited by R. A. Lessard and H. Franke, International Society for Optics and Photonics (SPIE, 1997) pp. $467-487$.

${ }^{10}$ L. Ferrighi, L. Frediani, C. Cappelli, P. Sałek, H. Ågren, T. Helgaker, and K. Ruud, "Density-functional-theory study of the electric-field-induced second harmonic generation (efishg) of push-pull phenylpolyenes in solution," Chem. Phys. Lett. 425, 267 - 272 (2006).

${ }^{11}$ J. F. McGilp, "A review of optical second-harmonic and sum-frequency generation at surfaces and interfaces," J. Phys. D: Applied Physics 29, 1812-1821 (1996).

${ }^{12}$ S. J. B. Yoo, C. Caneau, R. Bhat, M. A. Koza, A. Rajhel, and N. Antoniades, "Wavelength conversion by difference frequency generation in algaas waveguides with periodic domain inversion achieved by wafer bonding," App. Phys. Lett. 68, 2609-2611 (1996).

${ }^{13}$ D. Burland, "Optical nonlinearities in chemistry: Introduction," Chem. Rev. 94 (1994).

${ }^{14}$ M. Bass, P. A. Franken, J. F. Ward, and G. Weinreich, "Optical rectification," Phys. Rev. Lett. 9, 446-448 (1962).

${ }^{15}$ P. A. Franken, A. E. Hill, C. W. Peters, and G. Weinreich, "Generation of optical harmonics," Phys. Rev. Lett. 7, 118-119 (1961).

${ }^{16}$ B. J. Coe, L. A. Jones, J. A. Harris, B. S. Brunschwig, I. Asselberghs, K. Clays, and A. Persoons, "Highly unusual effects of $\pi$-conjugation extension on the molecular linear and quadratic nonlinear optical properties of ruthenium(ii) ammine complexes," J. Am. Chem. Soc. 125, 862-863 (2003).

${ }^{17}$ L. M. G. Abegão, R. D. Fonseca, F. A. Santos, J. J. Rodrigues, K. Kamada, C. R. Mendonça, S. Piguel, and L. De Boni, "First molecular electronic hyperpolarizability of series of $\pi$-conjugated oxazole dyes in solution: an experimental and theoretical study," RSC Adv. 9, 26476-26482 (2019).

${ }^{18} \mathrm{P}$. C. Ray, "The effects of $\pi$-conjugation on first hyperpolarizabilities of charged nlo chromophores," Chem. Phys. Lett. 394, 354 - 360 (2004).

${ }^{19}$ G. Bourhill, J.-L. Bredas, L.-T. Cheng, S. R. Marder, F. Meyers, J. W. Perry, and B. G. Tiemann, "Experimental demonstration of the dependence of the first hyperpolarizability of donor-acceptor-substituted polyenes on the ground-state polarization and bond length alternation,' J. Am. Chem. Soc. 116, 2619-2620 (1994).

${ }^{20}$ B. Champagne, D. Jacquemin, J.-M. André, and B. Kirtman, "Ab initio coupled hartreefock investigation of the static first hyperpolarizability of model all-trans-polymethineimine oligomers of increasing size," J. Phys. Chem. A 101, 3158-3165 (1997).

${ }^{21}$ N. A. Murugan, J. Kongsted, Z. Rinkevicius, and H. Ågren, "Breakdown of the first hyperpolarizability/bond-length alternation parameter relationship," Proc. Natl. Acad. Sci. U.S.A 107, 16453-16458 (2010).

${ }^{22}$ J. N. Woodford, M. A. Pauley, and C. H. Wang, "Solvent dependence of the first molecular hyperpolarizability of p-nitroaniline revisited,' J. Phys. Chem. A 101, 1989-1992 (1997).

${ }^{23}$ M. M. Alam, V. Kundi, and P. P. Thankachan, "Solvent effects on static polarizability, static first hyperpolarizability and one- and two-photon absorption properties of functionalized triply twisted möbius annulenes: a dft study," Phys. Chem. Chem. Phys. 18, 21833-21842 (2016).

${ }^{24} \mathrm{~B}$. Orr and J. Ward, "Perturbation theory of the non-linear optical polarization of an isolated system," Mol. Phys. 20, 513-526 (1971).

${ }^{25}$ J. L. Oudar, "Optical nonlinearities of conjugated molecules. stilbene derivatives and highly polar aromatic compounds," J. Chem. Phys. 67, 446457 (1977).

${ }^{26}$ J. L. Oudar and D. S. Chemla, "Hyperpolarizabilities of the nitroanilines and their relations to the excited state dipole moment," J. Chem. Phys. 66 , 2664-2668 (1977). 
${ }^{27}$ D. M. Bishop, "Explicit nondivergent formulas for atomic and molecular dynamic hyperpolarizabilities," J. Chem. Phys. 100, 6535-6542 (1994).

${ }^{28}$ D. M. Bishop, B. Kirtman, and B. Champagne, "Differences between the exact sum-over-states and the canonical approximation for the calculation of static and dynamic hyperpolarizabilities," J. Chem. Phys. 107, $5780-$ 5787 (1997).

${ }^{29}$ B. Champagne and B. Kirtman, "Evaluation of alternative sum-over-states expressions for the first hyperpolarizability of push-pull $\pi$-conjugated systems," J. Chem. Phys. 125, 024101 (2006).

${ }^{30}$ M. G. Kuzyk, "Compact sum-over-states expression without dipolar terms for calculating nonlinear susceptibilities,” Phys. Rev. A 72, 053819 (2005).

${ }^{31} \mathrm{~W}$. Bartkowiak and R. Zaleśny, "Sos methods in calculations of electronic nlo properties," in Non-Linear Optical Properties of Matter: From Molecules to Condensed Phases, edited by M. G. Papadopoulos, A. J. Sadlej, and J. Leszczynski (Springer Netherlands, Dordrecht, 2006) pp. 129-150.

${ }^{32}$ S. J. A. van Gisbergen, J. G. Snijders, and E. J. Baerends, "Calculating frequency-dependent hyperpolarizabilities using time-dependent density functional theory," J. Chem. Phys. 109, 10644-10656 (1998).

${ }^{33}$ J. Carmona-Espíndola, R. Flores-Moreno, and A. M. Köster, "Static and dynamic first hyperpolarizabilities from time-dependent auxiliary density perturbation theory," Int. J. Quantum Chem. 112, 3461-3471 (2012).

${ }^{34}$ P. Cronstrand, Y. Luo, and H. Ågren, "Generalized few-state models for two-photon absorption of conjugated molecules," Chem. Phys. Lett. 352 $262-269$ (2002)

${ }^{35}$ M. M. Alam, M. Chattopadhyaya, and S. Chakrabarti, "Solvent induced channel interference in the two-photon absorption process - a theoretical study with a generalized few-state-model in three dimensions," Phys. Chem. Chem. Phys. 14, 1156-1165 (2012).

${ }^{36}$ M. M. Alam, M. Chattopadhyaya, S. Chakrabarti, and K. Ruud, "Chemical control of channel interference in two-photon absorption processes," Acc. Chem. Res. 47, 1604-1612 (2014).

${ }^{37}$ M. M. Alam, M. T. P. Beerepoot, and K. Ruud, "Channel interference in multiphoton absorption,” J. Chem. Phys. 146, 244116 (2017).

${ }^{38}$ M. T. P. Beerepoot, M. M. Alam, J. Bednarska, W. Bartkowiak, K. Ruud, and R. Zaleśny, "Benchmarking the performance of exchange-correlation functionals for predicting two-photon absorption strengths," J. Chem. Theory Comput. 14, 3677-3685 (2018).

${ }^{39}$ M. M. Alam, M. Chattopadhyaya, S. Chakrabarti, and K. Ruud, "Highpolarity solvents decreasing the two-photon transition probability of through-space charge-transfer systems - a surprising in silico observation,' J. Phys. Chem. Lett. 3, 961-966 (2012).

${ }^{40}$ M. M. Alam, M. Chattopadhyaya, and S. Chakrabarti, "Enhancement of twist angle dependent two-photon activity through the proper alignment of ground to excited state and excited state dipole moment vectors,' J. Phys. Chem. A 116, 8067-8073 (2012).

${ }^{41}$ M. M. Alam, R. Misra, and K. Ruud, "Interplay of twist angle and solvents with two-photon optical channel interference in aryl-substituted bodipy dyes," Phys. Chem. Chem. Phys. 19, 29461-29471 (2017).

${ }^{42}$ P. Fischer, D. S. Wiersma, R. Righini, B. Champagne, and A. D. Buckingham, "Three-wave mixing in chiral liquids," Phys. Rev. Lett. 85, 4253-4256 (2000).

${ }^{43}$ J. O. Morley, "Nonlinear optical properties of organic molecules. xii. calculations of the hyperpolarizabilities of donor-acceptor polyynes," Int. J. Quantum Chem. 46, 19-26 (1993), https://onlinelibrary.wiley.com/doi/pdf/10.1002/qua.560460103.

${ }^{44}$ A. Willetts, J. E. Rice, D. M. Burland, and D. P. Shelton, "Problems in the comparison of theoretical and experimental hyperpolarizabilities," J. Chem. Phys. 97, 7590-7599 (1992)

${ }^{45}$ H. A. Kurtz and D. S. Dudis, "Quantum mechanical methods for predicting nonlinear optical properties," in Rev. Comp. Chem. (John Wiley \& Sons, Ltd, 2007) Chap. 5, pp. 241-279.

${ }^{46}$ A. D. Buckingham and D. A. Long, "Polarizability and hyperpolarizability [and discussion]," Philos. Trans. R. Soc. A 293, 239-248 (1979).

${ }^{47} \mathrm{H}$. Reis, "Problems in the comparison of theoretical and experimental hyperpolarizabilities revisited," J. Chem. Phys. 125, 014506 (2006).

${ }^{48}$ M. J. Frisch, G. W. Trucks, H. B. Schlegel, G. E. Scuseria, M. A. Robb J. R. Cheeseman, G. Scalmani, V. Barone, G. A. Petersson, H. Nakatsuji, X. Li, M. Caricato, A. V. Marenich, J. Bloino, B. G. Janesko, R. Gomperts, B. Mennucci, H. P. Hratchian, J. V. Ortiz, A. F. Izmaylov, J. L.
Sonnenberg, D. Williams-Young, F. Ding, F. Lipparini, F. Egidi, J. Goings, B. Peng, A. Petrone, T. Henderson, D. Ranasinghe, V. G. Zakrzewski, J. Gao, N. Rega, G. Zheng, W. Liang, M. Hada, M. Ehara, K. Toyota R. Fukuda, J. Hasegawa, M. Ishida, T. Nakajima, Y. Honda, O. Kitao, H. Nakai, T. Vreven, K. Throssell, J. A. Montgomery, Jr., J. E. Peralta, F. Ogliaro, M. J. Bearpark, J. J. Heyd, E. N. Brothers, K. N. Kudin, V. N. Staroverov, T. A. Keith, R. Kobayashi, J. Normand, K. Raghavachari, A. P. Rendell, J. C. Burant, S. S. Iyengar, J. Tomasi, M. Cossi, J. M. Millam, M. Klene, C. Adamo, R. Cammi, J. W. Ochterski, R. L. Martin, K. Morokuma, O. Farkas, J. B. Foresman, and D. J. Fox, "Gaussian16 Revision C.01," (2016), gaussian Inc. Wallingford CT.

${ }^{49}$ P. Sałek, O. Vahtras, T. Helgaker, and H. Ågren, "Density-functional theory of linear and nonlinear time-dependent molecular properties," J. Chem. Phys. 117, 9630-9645 (2002).

${ }^{50}$ K. Aidas, C. Angeli, K. L. Bak, V. Bakken, R. Bast, L. Boman, O. Christiansen, R. Cimiraglia, S. Coriani, P. Dahle, E. K. Dalskov, U. Ekström, T. Enevoldsen, J. J. Eriksen, P. Ettenhuber, B. Fernández, L. Ferrighi, H. Fliegl, L. Frediani, K. Hald, A. Halkier, C. Hättig, H. Heiberg, T. Helgaker, A. C. Hennum, H. Hettema, E. Hjertenæs, S. Høst, I-M. Høyvik, M. F. Iozzi, B. Jansík, H. J. A. Jensen, D. Jonsson, P. Jørgensen, J. Kauczor, S. Kirpekar, T. Kjærgaard, W. Klopper, S. Knecht, R. Kobayashi, H. Koch, J. Kongsted, A. Krapp, K. Kristensen, A. Ligabue, O. B. Lutnæs, J. I. Melo, K. V. Mikkelsen, R. H. Myhre, C. Neiss, C. B. Nielsen, P. Norman, J. Olsen, J. M. H. Olsen, A. Osted, M. J. Packer, F. Pawlowski, T. B. Pedersen, P. F. Provasi, S. Reine, Z. Rinkevicius, T. A. Ruden, K. Ruud, V. V. Rybkin, P. Sałek, C. C. M. Samson, A. S. de Merás, T. Saue, S. P. A. Sauer, B. Schimmelpfennig, K. Sneskov, A. H. Steindal, K. O. Sylvester-Hvid, P. R. Taylor, A. M. Teale, E. I. Tellgren, D. P. Tew, A. J. Thorvaldsen, L. Thøgersen, O. Vahtras, M. A. Watson, D. J. D. Wilson, M. Ziolkowski, and H. Ågren, "The dalton quantum chemistry program system," WIRES Comput. Mol. Sci. 4, 269-284 (2014).

51 "Lsdalton, a linear scaling molecular electronic structure program," (2018), release v2018.0 (2018), see http://daltonprogram.org.

${ }^{52}$ M. M. A. Alam, M. T. P. Beerepoot, and K. Ruud, "Script for: A generalized few-state model for the first hyperpolarizability," Zenodo (2020), http://doi.org/10.5281/zenodo.3737523.

${ }^{53}$ W. Bartkowiak, W. Niewodniczański, T. Misiaszek, and R. Zaleśny, "Firstorder hyperpolarizability of pyridinium n-phenolate betaine dye: Ab initio study," Chem. Phys. Lett. 411, 8 - 13 (2005).

${ }^{54}$ R. Zaleśny, M. G. Papadopoulos, W. Bartkowiak, and A. Kaczmarek, "On the electron correlation effects on electronic and vibrational hyperpolarizability of merocyanine dyes," J. Chem. Phys. 129, 134310 (2008)

${ }^{55}$ R. Zaleśny, I. W. Bulik, W. Bartkowiak, J. M. Luis, A. Avramopoulos, M. G Papadopoulos, and P. Krawczyk, "Electronic and vibrational contributions to first hyperpolarizability of donor-acceptor-substituted azobenzene," J. Chem. Phys. 133, 244308 (2010).

${ }^{56}$ I. W. Bulik, R. Zaleśny, W. Bartkowiak, J. M. Luis, B. Kirtman, G. E. Scuseria, A. Avramopoulos, H. Reis, and M. G. Papadopoulos, "Performance of density functional theory in computing nonresonant vibrational (hyper)polarizabilities," J. Comp. Chem. 34, 1775-1784 (2013).

${ }^{57}$ A. Miniewicz, S. Bartkiewicz, E. Wojaczynska, T. Galica, R. Zalesny, and R. Jakubas, "Second harmonic generation in nonlinear optical crystals formed from propellane-type molecules," J. Mater. Chem. C 7, 1255-1262 (2019).

${ }^{58}$ B. Gao, M. Ringholm, R. Bast, K. Ruud, A. J. Thorvaldsen, and M. Jaszuński, "Analytic density functional theory calculations of pure vibrational hyperpolarizabilities: The first dipole hyperpolarizability of retinal and related molecules," J. Phys. Chem. A 118, 748-756 (2014).

${ }^{59}$ W.-q. Li, X. Zhou, Y. Chang, W. Quan Tian, and X.-D. Sun, "Temperature dependence of nonlinear optical properties in li doped nano-carbon bowl material," App. Phys. Lett. 102, 153307 (2013).

${ }^{60} \mathrm{M}$. de Wergifosse and B. Champagne, "Electron correlation effects on the first hyperpolarizability of push-pull $\pi$-conjugated systems," J. Chem. Phys. 134, 074113 (2011)

${ }^{61}$ B. Champagne, E. A. Perpète, S. J. A. van Gisbergen, E.-J. Baerends, J. G. Snijders, C. Soubra-Ghaoui, K. A. Robins, and B. Kirtman, "Assessment of conventional density functional schemes for computing the polarizabilities and hyperpolarizabilities of conjugated oligomers: An ab initio investigation of polyacetylene chains," J. Chem. Phys. 109, 10489-10498 (1998). 
${ }^{62}$ B. Kirtman, B. Champagne, and D. M. Bishop, "Electric field simulation of substituents in donoracceptor polyenes: a comparison with ab initio predictions for dipole moments, polarizabilities, and hyperpolarizabilities," J. Am. Chem. Soc. 122, 8007-8012 (2000).

${ }^{63}$ D. Jacquemin, B. Champagne, and C. Hättig, "Correlated frequencydependent electronic first hyperpolarizability of small push-pull conjugated chains," Chem. Phys. Lett. 319, 327-334 (2000).

${ }^{64}$ R. Orlando, V. Lacivita, R. Bast, and K. Ruud, "Calculation of the first static hyperpolarizability tensor of three-dimensional periodic compounds with a local basis set: A comparison of lda, pbe, pbe0, b3lyp, and hf results," J. Chem. Phys. 132, 244106 (2010).

${ }^{65}$ R. Orlando, R. Bast, K. Ruud, U. Ekström, M. Ferrabone, B. Kirtman, and R. Dovesi, "The first and second static electronic hyperpolarizabilities of zigzag boron nitride nanotubes. an ab initio approach through the cou- pled perturbed kohn-sham scheme,” J. Phys. Chem. A 115, 12631-12637 (2011)

${ }^{66}$ L. Frediani, H. Ågren, L. Ferrighi, and K. Ruud, "Second-harmonic generation of solvated molecules using multiconfigurational self-consistent-field quadratic response theory and the polarizable continuum model," J. Chem. Phys. 123, 144117 (2005).

${ }^{67}$ P. Norman, B. Schimmelpfennig, K. Ruud, H. J. A. Jensen, and H. Ågren, "Relativistic effects on linear and nonlinear polarizabilities studied by effective-core potential, douglas-kroll, and dirac-hartree-fock response theory," J. Chem. Phys. 116, 6914-6923 (2002).

${ }^{68}$ Y. Luo, D. Jonsson, P. Norman, K. Ruud, O. Vahtras, B. Minaev, H. Ågren, A. Rizzo, and K. V. Mikkelsen, "Some recent developments of high-order response theory,” Int. J. Quantum Chem. 70, 219-239 (1998). 


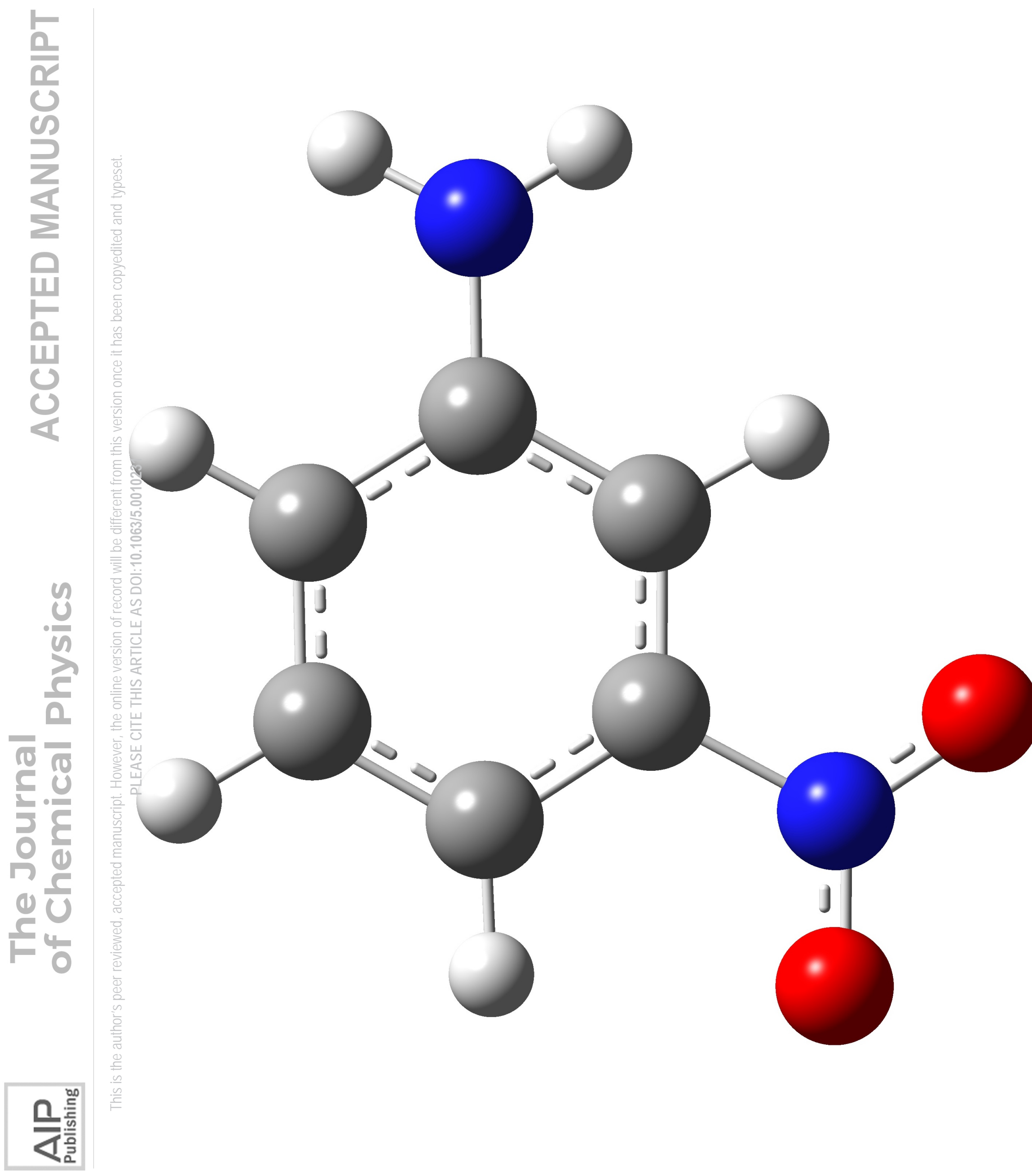




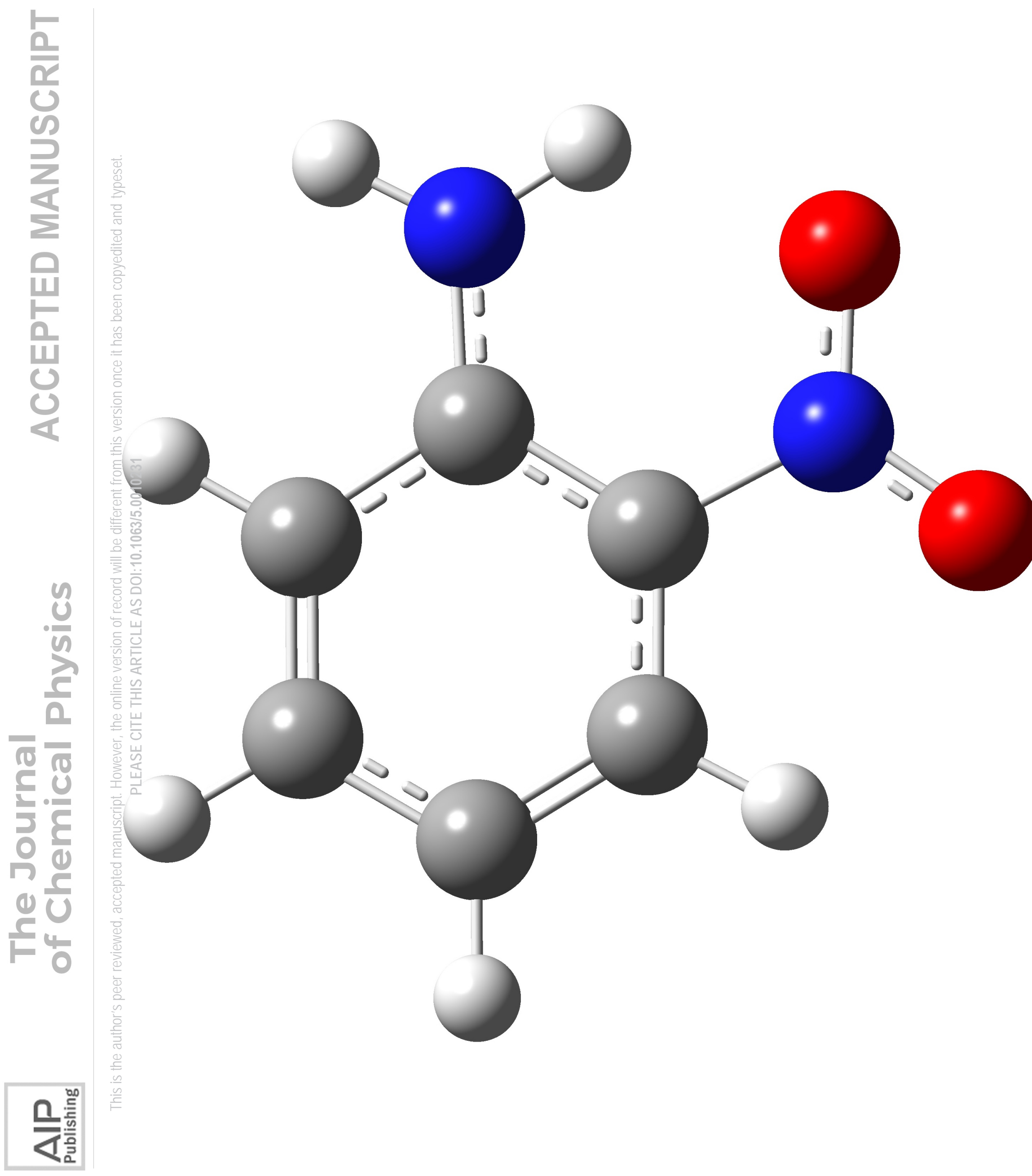



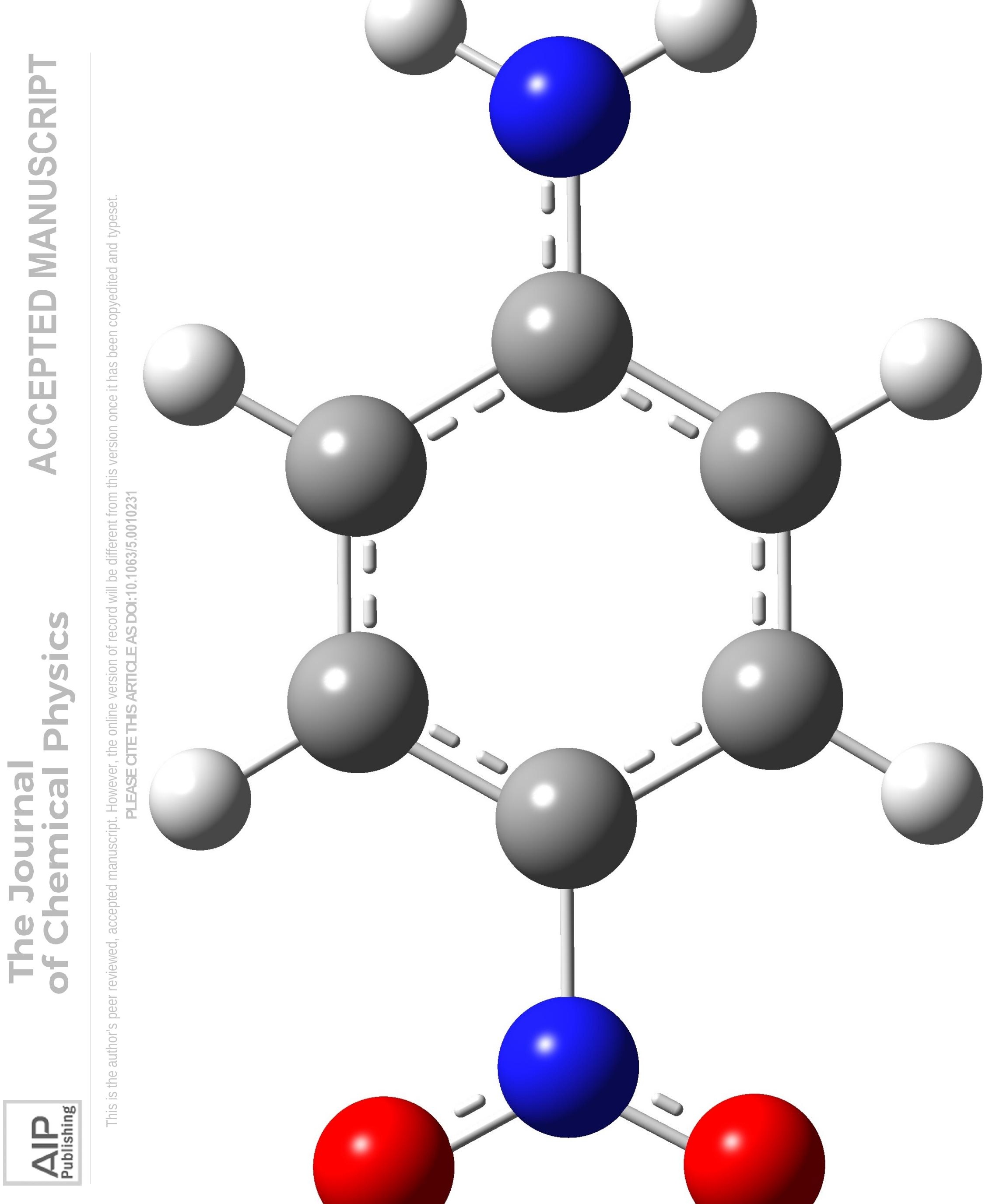


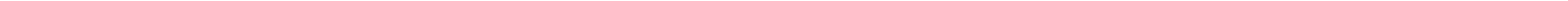




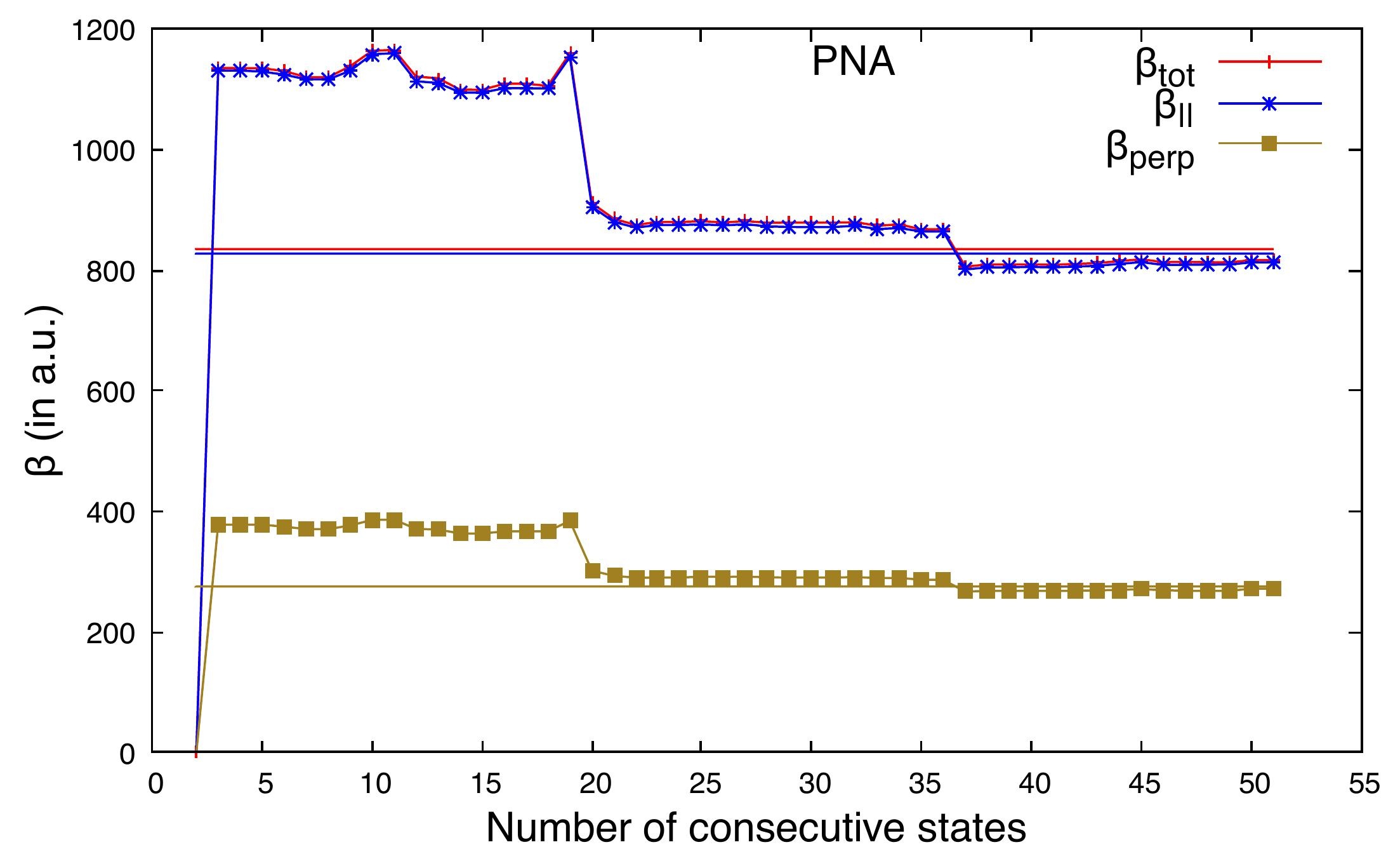




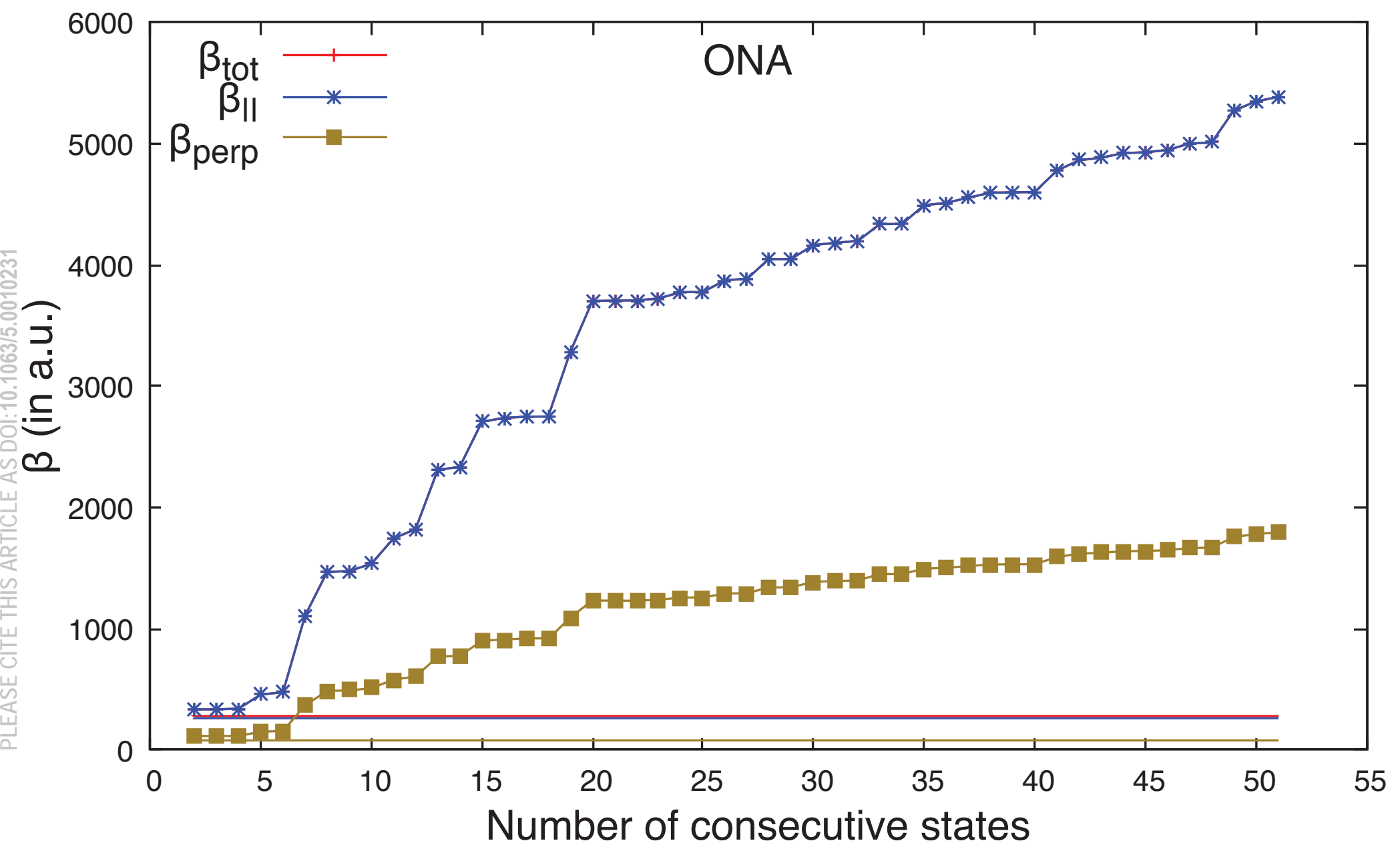




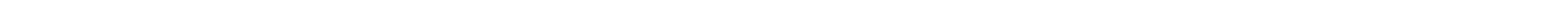




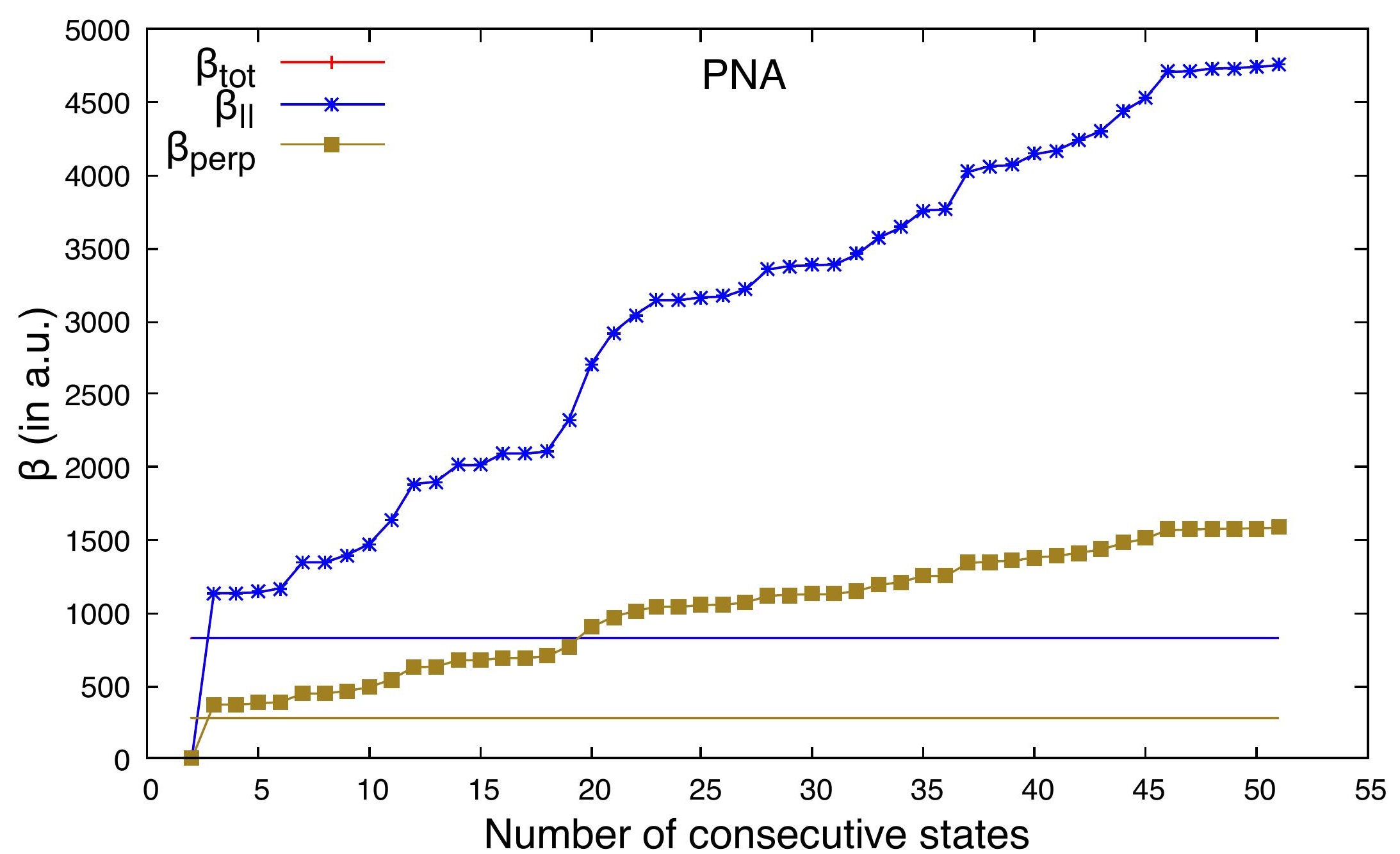

